\title{
The Hydrogen Atom Fundamental Resonance States
}

\author{
André Michaud \\ Service de Recherche Pédagogique, Québec, Canada \\ Email: srp2@srpinc.org
}

How to cite this paper: Michaud, A. (2018) The Hydrogen Atom Fundamental Resonance States. Journal of Modern Physics, 9, 1052-1110.

https://doi.org/10.4236/jmp.2018.95067

Received: March 27, 2018

Accepted: April 25, 2018

Published: April 28, 2018

Copyright (c) 2018 by author and Scientific Research Publishing Inc. This work is licensed under the Creative Commons Attribution International License (CC BY 4.0).

http://creativecommons.org/licenses/by/4.0/

(c) (i) Open Access

\begin{abstract}
In the 1920's, Louis de Broglie's observation that the integer sequence that could be related to the interference patterns produced by the various electromagnetic energy quanta emitted by hydrogen atoms was identical to those of very well known classical resonance processes, made him conclude that electrons were captive in resonance states within atoms. This led Schrödinger to propose a wave function to represent these resonance states that still have not been reconciled with the electromagnetic properties of electrons. This article is meant to identify and discuss the electromagnetic harmonic oscillation properties that the electron must possess as a resonator in order to explain the resonance volume described by the wave function, as well as the electromagnetic interactions between the elementary charged particles making up atomic structures that could explain electronic and nucleonic orbitals stability. An unexpected benefit of the expanded space geometry required to establish these properties and interactions is that the fundamental symmetry requirement is respected by structure for all aspects of the distribution of energy within electromagnetic quanta.
\end{abstract}

\section{Keywords}

Wave Function, Electron Resonance States, Elementary Electromagnetic Particles, Electromagnetism, Hydrogen Atom

\section{Introduction}

This paper does not propose an alternate approach to quantum mechanics, but rather an addition to the already established descriptions of orbital resonance states provided by Schrödinger's wave function, Heisenberg's statistical distribution and Feynman's path integral, involving a clear description of the electro- 
magnetic resonators responsible for the establishment of the related resonance volumes, meant to lay the groundwork for the eventual establishment of more elaborate wave functions that will for the first time completely account for the electromagnetic nature of these resonators.

The detailed mathematical proof of complete conformity with electromagnetism and with every aspect of all experimental data on record is provided in a series of articles previously published that are given in reference as required. This new approach is in complete agreement with the methods of QED and QFT and complements them by clarifying the function of the magnetic aspect of the energy of which electromagnetic elementary particles and their carrying energy are made, in a manner that allows describing their permanently localizable self-sustaining internal electromagnetic structure.

The key concept that triggered this particular research is an aspect of the wave function that seems to have escaped general attention almost from the moment that Quantum Mechanics (QM) was established to represent the hydrogen ground state from the correlation of Heisenberg's statistical representation and Schrödinger's wave function. This concerns the very reason why Schrödinger came up with the idea of using a wave function to describe the already well known stable ground state of the electron in the hydrogen atom. Strangely, it seems that the seminal paper which is at the origin of this major discovery never was translated to English [1].

This paper, written by Louis de Broglie, relates the interference patterns produced by the various electromagnetic energy frequencies emitted by hydrogen atoms to possible resonance states of the electron on what was then perceived as the various orbits that it could occupy in the hydrogen atom.

Here is de Broglie's description in his own words of the observation that led him in 1923 to this major conclusion, followed by its apparently first time ever translation to English:

"L'apparition, dans les lois du mouvement quantifié des électrons dans les atomes, de nombres entiers, me semblait indiquer Pexistence pour ces mouvements dinterférences analogues à celles que Pon rencontre dans toutes les branches de la théorie des ondes et où interviennent tout naturellement des nombres entiers."([2], p. 461).

"The occurrence of integers, in the laws of quantified motion of electrons in atoms seemed to me indicative of the existence for these motions of interferences analogous to those met in all branches of waves theory, where integers naturally occur."

Shortly afterwards, he published a note in the "Comptes rendus de P Académie des Sciences" in which he was proposing the first preliminary interpretation of the conditions that might explain the stability of the electron within atomic structures [1].

The critical conclusion of this note is the following. Original in French:

"Ponde de fréquence $v$ et de vitesse $c / \beta$ doit être en résonance sur la longueur de la trajectoire. Ceci conduit à la condition:" 
'the wave of frequency $v$ and velocity $c / \beta$ must be in resonance on the whole length of the trajectory. This leads to condition:"

$$
\frac{m_{o} \beta^{2} c^{2}}{\sqrt{1-\beta^{2}}} T_{r}=n h \quad n \text { being an integer }
$$

which is the stability condition determined by Bohr and Sommerfeld for a trajectory being run at constant velocity [1].

The following year, de Broglie published two more notes, in one of which he mentioned that from this viewpoint, Bohr's famous "frequency condition law" could be interpreted as involving some sort of "beat" of "pulsation" ("un battement" in the original French text), that is, a resonance state associating the frequency of the emitted wave to the initial electron stationary state and to its final stationary state [3] [4] and ([2], p. 462). Two years later Schrödinger introduced the wave function to account for this measurable condition.

The obvious starting point of his exploration was a complex-valued simple harmonic oscillation formula that then evolved into more elaborate spherical formulations to describe the ground state orbital of the hydrogen atom.

To this author's knowledge, no subsequent mention of the fact that Schrödinger's wave function is meant to describe a stable resonance state into which the localized electron remains captive can be found in the historical formal literature, except in a book published in 1953 to which the actual discoverers of Wave Mechanics and Quantum Mechanics collaborated [2].

Moreover, although Einstein contributed the text of the introduction of this book in German, and that Schrödinger contributed in English the Chapter that he provided, both contributions being translated to French on the facing pages, the remainder of the book was in French only. It also appears that this particularly important book, to which Einstein, Schrödinger, Pauli, Rosenfeld, Heisenberg, Yukawa, Davisson and de Broglie, to name only the most famous, and many more, jointly collaborated to provide a general overview of the state of quantum physics as of 1952, highlighting in historical context the contribution of Louis de Broglie, was apparently never translated to English nor to any other language to be made available to the international scientific community.

From what can be learned from this book, soon after the wave function was introduced by Schrödinger, whose validity was confirmed within a few years as being incontrovertibly related to resonance states, according to interference patterns generated during experiments carried out by Davisson and Germer, and also by G.P. Thompson ([2], p. 19), the adoption by the majority of researchers of Heisenberg's statistical representation, that replaces the volume of isotropic energy density defined by Schrödinger's wave function by a distribution of the energy density of the electron according to a statistical arrangement reflecting an "amplitude probability" perceived as being a more precise representation than the initial wave function, giving a greater density of energy presence in the vicinity of the Bohr radius for example, caused the fact that the wave function was initially meant to represent a resonance state to be obscured and neglected practi- 
cally from the onset.

The probabilistic interpretation also favored the idea of sudden jumps from one energy level to another, providing no mechanical explanation to these jumps, contrary to the wave equations that had the potential to allow descriptions of such changes as being mechanically progressive and mathematically describable processes, as re-emphasized by Schrödinger in 1953:

"To produce a coherent train of light waves of $100 \mathrm{~cm}$ length and more, as is observed in fine spectral lines, takes a time comparable with the average interval between transitions. The transition must be coupled with the production of the wave train... For the emitting system is busy all the time in producing the trains of light waves, it has no time left to tarry in the cherished "stationary states", except perhaps in the ground state." ([2], p. 18).

Even Einstein, who, like de Broglie and Schrödinger, was convinced that the electron remains constantly localized as it moves and always follows a precise trajectory, was unconvinced by the discovery by de Broglie of the relation between discrete quantum states and resonance states, presumably because he did not associate the concept of "mass" to electromagnetism in the same manner as de Broglie and Schrödinger.

Here is Einstein's comment in this regard that appears at the beginning of the introduction of this book. Original text in German:

"Ich will dem zusammen mit Frau B. Kaufman verfassten Beitrag zu diesem Bande einige Worte vorausschicken in der einzigen Sprache, in der ich mich mit einige Leichtigkeit ausdrücken kann. Es sind Worte der Entschuldigung. Sie sollen zeigen, warum ich, trotzdem ich De Broglie visionäre Entdeckung des inneren Zusammenhanges zwischen discreten Quantenzuständen und Resonanzzuständen in relativ jungen Jahren bewundernd miterlebt habe, doch unablässig nach einem Wege gesucht habe, das Quantenrätsel auf anderem Wege zu lösen oder doch wenigstens eine Lösung vorbereiten zu helfen.” ([2], p. 4).

"I will begin my contribution prepared for this book in collaboration with Mrs. Kaufman with a few words in the only language in which I can express myself with any ease. They are words to express regret. They are meant to show why-although I observed admiringly in my years of relative youth the genial discovery by Louis de Broglie of the intimate relation between the discrete quantum states and resonance states-I nevertheless ceaselessly searched for some manner to resolve the enigma of quanta by some other means, or at least help in preparing such a solution."

It turns out that Schrödinger and de Broglie were initially analyzing these observed resonance states in view of establishing a progressive mechanical explanation to the transitions between the stationary states, that would explain the generation of the bremsstrahlung photons responsible for the fine spectral lines detected in relation with these transitions, but that the immediate popularity of Heisenberg's statistical method in the community caused all research in this direction to be stalled almost from the start.

Schrödinger clearly expressed his frustration for the neglect of any research in 
this direction in the chapter that he contributed:

"For it must have given to de Broglie the same shock and disappointment as it gave to me, when we learnt that a sort of transcendental, almost psychical interpretation of the wave phenomenon had been put forward, which was very soon hailed by the majority of leading theorists as the only one reconcilable with experiment, and which has now become the orthodox creed, accepted by almost everybody, with a few notable exceptions." ([2], p. 16).

Schrödinger and de Broglie were obviously convinced that the frequency of an emitted quantum could be produced only by means of a progressive mechanical process dependent on the resonance characteristics of an electron initial stationary state, and whose emission mechanically determined in a clearly describable manner the altered resonance characteristics of the final stationary states and that solving this problem would be useful not only in spectroscopy, but also in chemistry.

It seems that Schrödinger's frustration was well justified, considering that it took 55 years after he so openly aired this protest in this book and also in a paper titled "Are there quantum jumps" published the same year in the "British Journal for the Philosophy of Science" [5], that is, 80 years after he introduced the wave function, for the first hints of renewed interest in resonance states in relation with the wave function to reappear in the community. This recent analysis can be found in a paper by V. A. Golovko [6] published in 2008 .

The outcome of the adoption by the majority of theorists of the statistical method as representing fundamental reality then led to the establishment of the quantum field theory (QFT) grounded on an axiomatic fundamental concept of spontaneous quantum energy fluctuations on either side of a zero point energy level that would exist everywhere in space, that establishes virtual photons (bosons) as being the force carriers that would explain the energy levels and motion of real elementary electromagnetic particles in space.

These hypothetical spontaneous stochastic fluctuations of the underlying quantum field are also considered as explaining an apparently erratic transverse quivering motion observed in the behavior of moving electrons in certain circumstances that Schrödinger named "Zitterbewegung", which we will analyze further on [7].

It is quite obvious that QFT is correctly grounded on Maxwell's electromagnetic wave theory and equations, but it nevertheless obscures the fact that in electromagnetism, an electron, for example, which is electrically charged, can be made to move in a straight line when immersed in equal density ambient $E$ and $\boldsymbol{B}$ fields; that if these intensities are made to simultaneously gradually vary, even if the variation is infinitesimally progressive, its velocity will vary just as gradually, and if their relative densities are made to gradually differ from each other, this will cause the electron to just as gradually curve its trajectory, which are processes all aspects of which can be calculated and controlled with the Lorentz equation $(\boldsymbol{F}=q(\boldsymbol{E}+\boldsymbol{v} \times \boldsymbol{B}))$.

This behavior of electrons completely validates the possibility that if the QFT 
concept of virtual bosons being the force carrier was replaced by the infinitesimally progressive Coulomb interaction stemming from Maxwell's first equation, that is, Gauss's equation for the electric field, this opens up the possibility that electromagnetic bremsstrahlung photons could be defined as self-sustaining their own motion in a localized manner without the need for any underlying ether, from the simple interaction of their own internal mutually inducing $E$ and B fields in conformity with Maxwell's grounding hypothesis, and that they could be defined as default self-guiding in straight line from the default equal densities of their own internal $\boldsymbol{E}$ and $\boldsymbol{B}$ fields [8].

\section{The $E$ and $B$ Fields of the Moving Electron}

It can also be observed that the resonance states of the electron are not the only aspects of electrons that seems to have been the object of little research over the course of the past century.

Despite the known facts that the electron possesses and electric charge, that it can be guided by progressively varying ambient electric $\boldsymbol{E}$ and magnetic $\boldsymbol{B}$ fields and that the "wave" aspect of its established "wave-particle" nature confirm that it is an electromagnetic particle, it seems that the intrinsic $E$ and $B$ fields of the electron itself, that is the $\boldsymbol{E}$ and $\boldsymbol{B}$ fields that must be associated to its very charge and mass, have apparently not yet been investigated in the community.

Indeed, the only relations between the electron and $E$ and $B$ fields that can apparently be found in the literature of the past hundred years specifically refer to the motion of electrons in ambient electric or magnetic fields, without any mention of any kind of interaction between these external fields and those that have by structure to be related to the electric charge and rest mass of the electron.

The first breakthrough in this direction is fairly recent. In 2003, Paul Marmet succeeded in directly relating the increasing magnetic field of an accelerating electron to its increase in relativistic mass from quantizing the electron charge in the Biot-Savart equation [9].

After he established the electron charge as remaining invariant at its unit value (1.602176462E-19C) in the Biot-Savart equation, his equation 17 now provides us with an electromagnetic equation that allows directly calculating the mass increment corresponding to the magnetic field increment of the accelerating electron:

$$
\Delta m_{m}=\frac{\mu_{0}\left(e^{-}\right)^{2}}{8 \pi r_{e}} \frac{v^{2}}{c^{2}}
$$

This equation thus directly associates the concept of "classical mass" to the real electromagnetic energy that must by definition be associated with this "magnetic field" increment of the electron in motion, which involves by similarity that the intrinsic magnetic field of the electron must also be associated to the real electromagnetic energy making up its invariant rest mass, as we will shortly see. 
He also observed that since the varying inertial mass of the moving electron is given by

$$
m=\gamma m_{e}
$$

and that the Lorentz $\gamma$ factor can be expanded as the following series:

$$
\gamma=1+\left\{\frac{1 v^{2}}{2 c^{2}}+\frac{3 v^{4}}{8 c^{4}}+\frac{5 v^{6}}{16 c^{6}}+\frac{35 v^{8}}{128 c^{8}}+\cdots\right\}
$$

and that the term $(v / c)^{4}$ and other higher order terms are negligible with respect to the $(v / c)^{2}$ term, they can be ignored for low relativistic velocities, which allows establishing the following equality from Equation (4):

$$
\gamma-1=\frac{1}{2} \frac{v^{2}}{c^{2}}
$$

Knowing that the momentum related relativistic kinetic energy of a moving electron is obtained from the following standard equation, which makes use of the right term of Equation (5):

$$
\Delta K=m_{0} c^{2}(\gamma-1)
$$

we can similarly calculate the relativistic mass increment from combining Equation (3) and Equation (5):

$$
\Delta m=m-m_{e}=m_{e}(\gamma-1)=\frac{m_{e}}{2} \frac{v^{2}}{c^{2}}
$$

Comparing now Equation (2) with Equation (7), we observe that we now have two different equations representing the same mass increment of the moving electron; that is, Equation (2) providing this increment as the mass of the magnetic field increment, while Equation (7) provides this same increment as a "classical mass" increment. We can thus equate Equations (2) and (7) in the following manner:

$$
\Delta m_{m}=\Delta m=\frac{\mu_{0}\left(e^{-}\right)^{2}}{8 \pi r_{e}} \frac{v^{2}}{c^{2}}=\frac{m_{e}}{2} \frac{v^{2}}{c^{2}}
$$

and finally, when the velocity becomes infinitesimal, both velocities ratios can be ignored to finally reveal the surprising fact that the mass of the intrinsic magnetic energy of the electron makes up exactly half of its invariant rest mass, which is the critically important conclusion reached by Marmet:

$$
m_{m}=\frac{\mu_{0} e^{2}}{8 \pi r_{e}}=\frac{m_{e}}{2}
$$

\section{The Electron Carrying Energy}

Let us consider for a moment the meaning of $\Delta m_{m}$ from Equation (2) and of $\Delta K$ from Equation (6). To be able to really see what is involved, let us use the well known concrete case of the electron relativistic velocity of $2,187,647.561 \mathrm{~m} / \mathrm{s}$ on the theoretical classical Bohr ground state orbit. Using this velocity to resolve Equation (2), we obtain the following mass increment: 


$$
\Delta m_{m}=\frac{\mu_{0}\left(e^{-}\right)^{2}}{8 \pi r_{e}} \frac{v^{2}}{c^{2}}=2.425337715 \mathrm{E}-35 \mathrm{~kg}
$$

which is the magnetic field mass increment to be added to the rest mass of the electron to obtain the total effective electron mass that experimentalists have to deal with when transversally deflecting electrons moving freely at this corresponding relativistic velocity of $2,187,647.561 \mathrm{~m} / \mathrm{s}$.

Multiplying now this value by $c^{2}$, we obtained the energy in joules constituting this amount of mass $(2.179784832 \mathrm{E}-18 \mathrm{j})$, and further dividing this value in joules by the unit charge of the electron (1.602176462E-19C), we obtain its conversion to electronvolts $(13.6 \mathrm{eV})$.

Let us now calculate with Equation (6) the momentum kinetic energy related to this same velocity of the electron:

$$
\Delta K=m_{0} c^{2}(\gamma-1)=2.179784832 \mathrm{E}-18 \mathrm{j}
$$

If we now divide this value by the unit charge of the electron, we again obtain a value in electronvolts equal to $(13.6 \mathrm{eV})$.

So, we observe that both $\Delta K$ and $\Delta m_{m}$ resolve to the same $13.6 \mathrm{eV}$ energy value, that we may be strongly tempted to consider as representing the same energy quantum calculated by different means.

But, it can hardly be disputed that on one hand, $\Delta m_{m}$ measures the energy contained in a mass increment corresponding to an increase of the global magnetic field of the electron and that on the other hand, $\Delta K$ measures the well known kinetic energy that propels the effective mass of the electron at the stated velocity, an effective mass that includes by structure the quantity $\Delta m_{m}$ calculated with Equation (2) on top of including the invariant rest mass of the electron.

Consequently, the only possible conclusion is that these two instances of 13.6 $\mathrm{eV}$ are different and are both induced simultaneously in the electron at this velocity, and consequently are in reality two "half-quanta" of energy whose sum constitutes a single quantum of "carrying energy" that exists separately from the energy quantum of which the electron invariant rest mass is made, one of which converting to a mass increment, while the other remains vectorially unidirectional, propelling the total effective mass of the electron at the stated velocity.

All calculations with Equations (2) and (6) for any velocity will reveal that this even split between an amount going into an increase in magnetic field mass and a translational momentum related amount of kinetic energy is maintained for the whole range of all possible relativistic velocities.

Interestingly, the total amount of $27.2 \mathrm{eV}$ that results from adding the energy of the magnetic mass increment obtained from Equation (2) and the momentum energy obtained from Equation (6), is exactly equal to the single amount of energy that can be calculated with the Coulomb equation as a function of the mean axial distance separating the electron ground state orbital from the hydrogen nucleus, and that corresponds to relativistic velocity $2,187,647.561 \mathrm{~m} / \mathrm{s}$ : 


$$
E=\int_{a_{0}}^{\infty} \frac{1}{4 \pi \varepsilon_{o}} \frac{e^{2}}{a_{0}^{2}} \cdot \mathrm{d} a_{0}=0-\frac{1}{4 \pi \varepsilon_{o}} \frac{e^{2}}{a_{0}}=-4.359743805 \mathrm{E}-18 \mathrm{~J}
$$

When dividing this amount of energy by the unit charge value (1.602176462E-19C), we effectively obtain in electronvolts the exact amount of energy obtained by summing up the energies obtained from Equations (2) and (6), that is, $27.2 \mathrm{eV}$, which confirms the validity of Equation (2) newly derived by Marmet, on top of confirming the fact that this total amount of energy induced for any relativistic velocity in a charged particle can be entirely obtained from an equation stemming from electromagnetism, that is, the Coulomb Equation (12), which now allows reuniting both $\Delta K$ and $\Delta m_{m}$ obtained from Equations (2) and (11) as belonging to a single quantum of energy now directly related to electromagnetism since they are simultaneously induced by the Coulomb force:

Charged particle carrying energy $=\Delta K+\Delta m_{m} c^{2}=4.359743805 \mathrm{E}-18 \mathrm{j}$

\section{The Issue of Momentum Energy Being Considered Conservative}

Examining Equation (13) now reveals a major disconnect between the traditional classical/relativistic mechanics concept of "momentum", that can be related only to the $\Delta K$ half of the energy adiabatically induced by the Coulomb force, which is deemed, from the traditional perspective, to reduce to zero when a body is not in motion, even if it remains adiabatically induced from the electromagnetic perspective, when the electron is captive in the hydrogen ground state orbital, in which it is now well understood that it is not moving on the theoretical Bohr orbit, as put in clear perspective in reference [10].

Moreover! There is no trace in traditional classical/relativistic mechanics, nor in traditional quantum mechanics, of the second component of Equation (13), that is, $\Delta m_{m} c^{2}$, which is adiabatically induced by the Coulomb force simultaneously with the $\Delta K$ component.

In classical/relativistic mechanics, momentum is obviously viewed as the most fundamental principle, a concept that was carried on to traditional quantum physics under the forms of the Hamiltonian and the Lagrangian. But in electromagnetism, the energy that sustains momentum is even more fundamental than momentum, given that it still remains adiabatically present by definition even when this momentum is inhibited, that is, even when an electrically charged particle, such as the electron, is stopped in its motion when captured in a state of axial electromagnetic equilibrium in one of the least action orbitals in an atom [10].

This fundamental disconnect between electromagnetism on one hand, and traditional classical mechanic, traditional relativistic mechanics and traditional quantum mechanics on the other, makes it all the more difficult to conceptually overcome, since the value of $\Delta K$ as calculated with Equation (11) depends uniquely on the "velocity" parameter, which means that if this velocity falls to zero, then no momentum, that is, no motion inducing kinetic energy is conceptually 
deemed to exist from the non-electromagnetism traditional perspectives, which is in flagrant contradiction with the fact that according to Equation (13) stemming from electromagnetism, this energy is adiabatically induced uniquely as a function of the axial distance between electrically charged particles by the Coulomb force, that forbids by very nature that any other level of energy be induced between two charges separated by this distance, which means that it can only remain induced even if the velocity of the particle is inhibited, as demonstrated in reference [10].

Even from the Quantum Mechanics perspective, the wave function accounts for the complete physical presence of this $13.6 \mathrm{eV} \Delta K$ momentum energy via the Hamiltonian even if it is experimentally established that the electron is unable to move toward the proton at any velocity despite the impossibility by structure that this momentum energy be vectorially oriented in any direction other than toward the proton.

This observation consequently brings to light the possibility that momentum kinetic energy can exist as a "material substance" irrespective of whether or not a forward velocity is involved, as analyzed in detail in references [8] [10] [11], and is at the heart of a new paradigm that now allows mechanically explaining a series of electromagnetic processes that find no explanation from the traditional conservative principles perspective [11] [12].

Having now made this connection, the analysis that follows will be carried on strictly from the electromagnetism perspective.

\section{Separating the Energy of the Magnetic Field Increment from That of the Magnetic Field of the Invariant Rest Mass of the Electron}

This new perspective now allows us to clearly separate the carrying energy of the electron from that of its rest mass and to calculate separately their electromagnetic frequencies and wavelengths with the standard equations $E=h v$ and $c=\lambda v$. We thus obtain the following frequency and electromagnetic wavelength for the $4.359743805 \mathrm{E}-18 \mathrm{j}$ reference carrying energy of the electron on the theoretical Bohr ground state orbit, which in fact corresponds to the mean carrying energy of the electron in the ground state orbital of the hydrogen atom:

$$
v=\frac{E}{h}=6.579683909 \mathrm{E} 15 \mathrm{~Hz}, \lambda=\frac{c}{v}=4.556335261 \mathrm{E}-08 \mathrm{~m}
$$

Similarly, we obtain the following frequency and electromagnetic wavelength for the energy of $E=m_{0} c^{2}=8.18710414 \mathrm{E}-14 \mathrm{j}$ making up the electron invariant rest mass, which wavelength is also known as the electron Compton wavelength:

$$
v=\frac{E}{h}=1.235589976 \mathrm{E} 20 \mathrm{~Hz}, \quad \lambda_{C}=\frac{c}{v}=2.426310215 \mathrm{E}-12 \mathrm{~m}
$$

We thus immediately observe that the energy related to the electron in motion involves the presence of not only one single simple harmonic electromagnetic 
oscillation, as the Schrödinger wave function seems to currently assume, but of two distinct harmonic oscillations, whose mutual resonance interaction is not yet clearly defined.

These values will be quite useful later on when the Zitterbewegung of the moving electron will be analyzed, as well as the complex electron resonance beat involving the interaction of these two harmonic oscillations with those of the proton inner electromagnetic elementary components when the electron is captive in the rest orbital of the hydrogen atom.

Let us also remark that although the concept of "wavelength" is sometimes assumed to represent a physical "length" to be associated to localized photons or even to the hypothetical electromagnetic waves of Maxwell's theory, such a wavelength can only be in reality a physical "distance" that such a localized photon or spread out theoretical electromagnetic wave needs to travel in space for one of the cycle of mutual induction of their electric and magnetic aspects to be completed in reference to their frequency.

Speaking of Maxwell's continuous electromagnetic wave concept, the experiments carried out by Huygens, Fresnel and Young that demonstrate that at the macroscopic level, when a macroscopic electromagnetic wavefront is made to meet a surface into which a small aperture is made, however small it may be from our macroscopic perspective, this small aperture becomes the source of a secondary spherical electromagnetic wavefront, which is often flaunted as "the proof" of the physical existence of continuous electromagnetic waves such as Maxwell conceived them.

There is a habit in the community of thinking of an "electromagnetic wavefront", but in reality there is an uninterrupted flow of electromagnetic energy in all of space, whether considered as a continuous wave phenomenon or as a crowd of countless separate point-like behaving electromagnetic photons that are constantly being individually emitted by de-exciting electrons in atoms, after these electrons were excited either out of atoms or just pushed further away from their nuclei to some metastable orbital.

In reality, this behavior of electromagnetic energy as measurable at the macroscopic level does not demonstrate any disconnect with the idea that this macroscopic electromagnetic wavefront could be made in reality of countless elementary point-like behaving electromagnetic photons that would interact, while moving through the small apertures, with the countless other point-like behaving electromagnetic elementary particles captive in various least action electromagnetic equilibrium states in the atoms making up the inner sides of the macroscopic apertures, and whose trajectories would consequently be deflected in such a way as to produce what seems to be, from our macroscopic perspective, "secondary spherical electromagnetic wavefronts" observed as they come out of the aperture.

There is absolutely nothing that rules out either the possibility that the individual photons emitted by de-exiting electrons in atoms all over the universe could continue behaving point-like after emission until they are subsequently 
absorbed by other charged particles, re-initiating in so doing the emission process, after having had their trajectories deflected numerous times before being absorbed by other charged particles at some other locations, as analyzed in reference [8].

Whether one concludes that electromagnetic energy really exists as a continuous wave phenomenon as perceived from our macroscopic level or that localized photons are the real thing at the submicroscopic level holds only to what a person has studied. Both schools of thought always had quite respectable adepts. The fact is that even if treating electromagnetic energy as localized quanta is consistent with the results of experiments carried out at the submicroscopic level, treating it as a continuous wave phenomenon remains consistent with the results of experiments carried out at the macroscopic level.

It seems however that the conclusion according to which this energy would physically exist as localized photons, as concluded by Planck, Einstein, de Broglie and Schrödinger, among others, allows clearer mechanical explanations of the various processes at the submicroscopic level.

\section{Particularities of Energy Calculation by Means of the Coulomb Equation}

Even though Equation (12) calculates the carrying energy of the electron at the hydrogen mean ground orbital distance from the central proton by mathematically accumulating this energy from "infinity" to this specific distance from $r=0$, it can be observed that this amount of energy can only be systematically equal to the actual amount of kinetic energy adiabatically induced by the Coulomb force as a function of this distance separating both electrical charges, a distance which is equal by structure to the distance separating point $d$ from point zero in the integration function (Figure 1).

It can be observed also that point zero of the integration function can be relocalized to the middle of the distance between both charges processed by the Coulomb equation without affecting energy calculation in any way, a central point $\otimes$ that will be later correlated with the central junction point of an expanded spatial geometry.

The method used by Marmet to derive Equation (9) from the Biot-Savart equation then allows deriving a new more general form of the Coulomb equation equivalent to traditional equation $E=h v$, that allows calculating the energy of any electromagnetic energy quantum without any need to use the Planck constant, and that also allows defining their intrinsic $\boldsymbol{E}$ and $\boldsymbol{B}$ fields strictly by means of a set of known electromagnetic constants.

Isolating the value of $m_{0}$ in Equation (9) established by Marmet and making use of the familiar equation $\mu_{0} \varepsilon_{0} c^{2}=1$ stemming from equivalent second partial derivatives of Maxwell's equations [13], that as far back as the 1860's allowed him to calculate the invariant speed of light from the two fundamental constants of vacuum $\varepsilon_{0}$ and $\mu_{0}$, will allow introducing the electrostatic permittivity constant of vacuum $\varepsilon_{0}$ to link up with the Coulomb equation. Isolating $\mu_{0}$ in this 


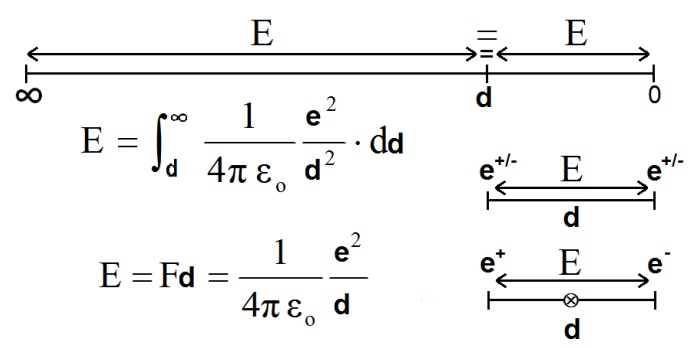

Figure 1. Energy equality between integration from infinity to distance " $\mathrm{d}$ " and between " $\mathrm{d}$ " and zero.

equation in the following manner $\mu_{0}=1 / \varepsilon_{0} c^{2}$ allows replacing it with its equivalent electromagnetic definition [14]:

$$
m_{0}=\frac{\mu_{0} e^{2}}{4 \pi r_{e}}=\frac{e^{2}}{4 \pi \varepsilon_{0} r_{0} c^{2}}
$$

Multiplying then both left and right terms of Equation (16) by $c^{2}$ will convert the equation from calculating mass to calculating energy, in this particular case, the energy quantum of which the invariant rest mass of the electron is made:

$$
E=m_{0} c^{2}=\frac{e^{2}}{4 \pi \varepsilon_{0} r_{0}}=8.18710414 \mathrm{E}-14 \mathrm{j}
$$

Assuming that $e^{2}$ is likely to represent any pair of charges in such a general equation, let us replace the "classical electron radius" $r_{0}$ used by Marmet by the classical radius of the Bohr theoretical orbit in the Bohr atom $a_{0}$ to remain coherent with the hydrogen atom example. Considering that the Coulomb force between two such charges requires involving the distance between the charges, let us further divide both sides of the equation by $a_{0}$ to finally obtain the equation allowing to calculate the Coulomb force, and to identify in the resulting equation the long established electrostatic constant whose exact value is $8.987551733 \mathrm{E}-9$ $\mathrm{Nm}^{2} / \mathrm{c}^{2}$, also known as the Coulomb constant:

$$
F=\frac{E}{r_{0}}=\frac{e^{2}}{4 \pi \varepsilon_{0} r_{0}^{2}} \text { where } \frac{1}{4 \pi \varepsilon_{0}}=k_{e}(\text { Coulomb constant })
$$

As a final validity confirmation, let us calculate the well-known Coulomb force that applies at the theoretical Bohr orbit, using the Bohr radius $a_{0}=$ $5.291772083 \mathrm{E}-11 \mathrm{~m}$ :

$$
F=\frac{e^{2}}{4 \pi \varepsilon_{0} a_{0}^{2}}=8.238721807 \mathrm{E}-08 \mathrm{~N}
$$

Now going back to Equation (17) that allows calculating the energy making up the electron rest mass, we observe that the only "possibly variable" parameter that determines the amount of energy of the quantum making up this mass is $r_{0}$, which is considered a fundamental constant known under the name of "the classical electron radius" and that Marmet used to derive Equation (9).

It is well understood in physics circles that despite its name, this constant cannot really be an actual "radius" of the electron, given that it is now well estab- 
lished experimentally that the electron behaves "point-like" in all scattering experiments. "Point-like behavior" meaning here that during all such scattering experiments, however energetic, no unbreachable limit was ever detected at some distance from electrons' centers, however close two electrons came to each others' centers.

So despite this unfortunate misleading misnomer, " $r_{0}$ " is nevertheless considered useful to define a "length", or "distance", not yet fully understood related to electromagnetic interactions involving electrons at the submicroscopic level.

But we may now have "telltales" as to what this "length" or "distance" may be, beginning with the observation that if we use it in Equation (12) instead of the classical Bohr radius $a_{0}$, we obtain the actual energy of the quantum of which the rest mass of the electron is made, as just calculated with the standard Equation (17), as if $r_{\mathrm{o}}$ was a really existing "distance" between a pair of still to be identified internal "would-be charges" involved in the still to be established electromagnetic inner oscillating structure of the electron, despite the fact that the "electric" charge of the electron is known to be unique and set at the fixed value of 1.602176462E-19C. Maybe some sort of point-like "charges" of some nature different from "electric" but that would still be acted upon by the Coulomb force, despite the strangeness of the idea.

We will see further on that such an internal structure has effectively been established involving a harmonic oscillation of the inner magnetic energy of the electron cyclically converting to two such "non-electric charges" and back to magnetic energy state. See Equation (53) further on.

One more telltale relates to the relation between $r_{0}$ and $\lambda_{c}$ that is, the electron Compton wavelength, that we just calculated with Equation (15). This further telltale relates to a relation between these two "constants" and the fine structure constant $\alpha$, first described in reference [15] in relation with the recall constant of Hooke's law as applied to the LC transverse oscillation of the magnetic energy of the rest mass of the electron, and which amounts to half the rest mass of the electron, as determined by Marmet with Equation (9).

From the calculation carried out in reference [14], the maximum transverse separation amplitude between these "charges" during this reciprocating LC oscillation would be exactly equal to $r_{0}=\alpha \lambda_{d} / 2 \pi$, which would amount to the maximum distance that these two still to be identified "non-electric would-be charges" would reach in space as they oscillate between this double-component state and the magnetic single component constituting the magnetic half of the energy of the electron invariant rest mass. This conclusion was confirmed later when the LC oscillating "neutrinic charges" of the electron were identified in reference [16]. This issue will be discussed further on.

This relation between $r_{0}, \lambda_{c}$ and $\alpha$ led to consider the possibility that the same method could be applied to calculate the energy of any self-sustaining electromagnetic quantum, and subsequent verification confirmed the possibility. It turns out that $r=\alpha \lambda / 2 \pi$ coincides with the maximum distance that two charges-either electric or neutrinic-can reach transversally during the LC os- 
cillation of any self-sustaining electromagnetic quantum during the reciprocating oscillation that causes them to cyclically induce the magnetic field of the particle as they close in on each other, and its regression as they move away from each other [15] [16] [17] [18] as we will see further on.

This means in fact that $r_{o}$ and $a_{o}$ are not really fundamental constants but only special cases of the whole range of possible electromagnetic energy transverse amplitudes coinciding with two quantized least action stable states of electromagnetic energy, that is, the invariant rest mass of the electron, and the least action electromagnetic equilibrium state of the electron in the hydrogen atom, and that they can be systematically replaced in the Coulomb equation by the more general variable expression " $\alpha \lambda / 2 \pi$ ", " $\lambda$ " being the electromagnetic longitudinal wavelength traditionally related to the electromagnetic energy quantum considered.

This allowed defining the following general equation in reference [14] by adapting Coulomb Equation (19) in the following manner (see also Figure 1):

$$
E=\int_{a_{0}}^{\infty} \frac{1}{4 \pi \varepsilon_{o}} \frac{e^{2}}{(\alpha \lambda / 2 \pi)^{2}} \cdot \mathrm{d} r=0-\frac{1}{4 \pi \varepsilon_{o}} \frac{e^{2} 2 \pi}{\alpha \lambda}=\frac{e^{2}}{2 \varepsilon_{o} \alpha \lambda}
$$

which defines an electromagnetic equation equivalent to $E=h v$, but that does not require the use of Planck's constant to calculate electromagnetic energy levels, whose complete derivation and justification was established in reference [14]:

$$
E=h v=\frac{e^{2}}{2 \varepsilon_{0} \alpha \lambda}
$$

One surprising benefit brought about by the establishment of this form of the Coulomb equation was that it finally allows unifying all classical force equations by allowing to reversibly derive the fundamental equation $F=m a$ from all of them [19], beside observing that the Coulomb equation is an integral part of the Biot-Savart equation, since it is derived from Marmet's derivation from the Biot-Savart equation.

\section{Separately Calculating the $E$ and $B$ Fields of the Electron and Those of Its Carrying Energy}

The development of Equation (21) then allowed separately defining in reference [14] the $\boldsymbol{E}$ and $\boldsymbol{B}$ fields equations accounting for the totality of the energy of which the invariant rest mass of the electron is made:

$$
\boldsymbol{B}=\frac{\mu_{0} \pi e c}{\alpha^{3} \lambda_{C}^{2}}=8.289000222 \mathrm{E} 13 \mathrm{~T} \text { and } \boldsymbol{E}=\frac{\pi e}{\varepsilon_{0} \alpha^{3} \lambda_{C}^{2}}=2.484979751 \mathrm{E} 22 \mathrm{~N} / \mathrm{C}
$$

and with the same equation, using the electromagnetic wavelength of its carrying energy, to calculate the $\boldsymbol{E}$ and $\boldsymbol{B}$ fields of this carrying energy. To remain consistent with our example of hydrogen atom ground state orbital, here are the $E$ and $\boldsymbol{B}$ fields calculated with the wavelength of the carrying energy obtained in Equation (14): 


$$
\boldsymbol{B}=\frac{\mu_{0} \pi e c}{\alpha^{3} \lambda^{2}}=235051.7341 \mathrm{~T} \text { and } \boldsymbol{E}=\frac{\pi e}{\varepsilon_{0} \alpha^{3} \lambda^{2}}=7.046673712 \mathrm{E} 13 \mathrm{~N} / \mathrm{C}
$$

Reference [14] then shows how magnetic and electric fields Equations (22) and (23) can be added to establish the combined $\boldsymbol{E}$ and $\boldsymbol{B}$ fields of the electron in motion. To remain consistent with the hydrogen atom ground state parameters, the wavelengths obtained with Equations (14) and (15) are used to calculate the corresponding fields:

$$
\begin{gathered}
\boldsymbol{B}=\frac{\pi \mu_{0} e c\left(\lambda^{2}+\lambda_{C}^{2}\right)}{\alpha^{3} \lambda^{2} \lambda_{C}^{2}}=8.289000246 \mathrm{E} 13 \mathrm{~T} \\
\boldsymbol{E}=\frac{\pi e}{\varepsilon_{0} \alpha^{3}} \frac{\left(\lambda^{2}+\lambda_{C}^{2}\right) \sqrt{\lambda_{C}\left(4 \lambda+\lambda_{C}\right)}}{\lambda^{2} \lambda_{C}^{2}\left(2 \lambda+\lambda_{C}\right)}=1.813341121 \mathrm{E} 13 \mathrm{~N} / \mathrm{C}
\end{gathered}
$$

It can now be confirmed that Equations (24) and (25) are valid by calculating with the values obtained, the well known relativistic velocity of the electron when moving with the $4.359743805 \mathrm{E}-18 \mathrm{j}$ reference energy of the hydrogen ground state $(27.2 \mathrm{eV})$ :

$$
v=\frac{\boldsymbol{E}}{\boldsymbol{B}}=\frac{1.813341121 \mathrm{E} 13}{8.289000246 \mathrm{E} 13} 10^{-7}=2187647.566 \mathrm{~m} / \mathrm{s}
$$

The reason why the result must be multiplied by $10^{-7}$, is that this factor, which was made part of the definitions of $\varepsilon_{o}$ and $\mu_{o}$ for these constants to remain in harmony with the CGS system when the MKS units were adopted [13], and that are part of the parameters required to calculate the $\boldsymbol{E}$ and $\boldsymbol{B}$ fields of the moving electron with Equations (24) and (25), ends up being squared in the denominator of the $\boldsymbol{E} / \boldsymbol{B}$ fraction of Equation (26), which is something not obvious unless actual calculations are carried out, as in our example. This unwanted squaring is circumvented by simply multiplying the equation by $10^{-7}$ during its resolution. See reference [13] for an explanation of why this factor must not be squared.

So we observe that the magnetic mass increment provided by Marmet's Equation ([9], Equation 17), which was previously reproduced as Equation (2), can be matched with a corresponding $\boldsymbol{B}$ field provided by Equation (23) from the electromagnetic wavelength of $4.556335256 \mathrm{E}-8 \mathrm{~m}$ of the corresponding quantum of energy (4.359743805E-18j), thus amending Equation (10) to obtain the mass increment using the velocity stemming from the $E$ and $B$ fields as calculated with Equation (26).

Given that Equation (26) provides the same relativistic velocity that Marmet established from the gamma factor [9] from Equations (4) and (5), and that he used in establishing Equation (2), the velocity term of Marmet's equation can be replaced by the $\boldsymbol{E} / \boldsymbol{B}$ relation that defines this velocity in Equation (26):

$$
\Delta m_{m}=\frac{\mu_{0}\left(e^{-}\right)^{2}}{8 \pi r_{e}} \frac{v^{2}}{c^{2}}=\frac{\mu_{0}\left(e^{-}\right)^{2}}{8 \pi r_{e}} \frac{(\boldsymbol{E} / \boldsymbol{B})^{2}}{c^{2}}=2.425337726 \mathrm{E}-35 \mathrm{~kg}
$$

thus allowing for the first time the calculation of a "classical mass" strictly from electromagnetic parameters. 
The magnetic energy density involved can now be established for the energy of the composite $\boldsymbol{B}$ field calculated with Equation (24):

$$
u_{B}=\frac{\boldsymbol{B}^{2}}{2 \mu_{0}}=\frac{1}{2 \mu_{0}}\left(\frac{\pi \mu_{0} e C}{\alpha^{3} \lambda^{2} \lambda_{C}^{2}}\right)^{2}\left(\lambda^{2}+\lambda_{C}^{2}\right)^{2}=2.733785559 E 33 \mathrm{j} / \mathrm{m}^{3}
$$

For comparison, here is the density of the magnetic field of the isolated invariant rest mass of the electron, making use of the invariant magnetic field of the electron calculated with Equation (22):

$$
u_{B}=\frac{B^{2}}{2 \mu_{0}}=\frac{1}{2 \mu_{0}}\left(\frac{\mu_{0} \pi e c}{\alpha^{3} \lambda_{C}^{2}}\right)^{2}=2.733785544 \mathrm{E} 33 \mathrm{j} / \mathrm{m}^{3}
$$

and that of the isolated carrying energy of the electron in the hydrogen ground state calculated with Equation (23) is:

$$
u_{B}=\frac{B^{2}}{2 \mu_{0}}=\frac{1}{2 \mu_{0}}\left(\frac{\mu_{0} \pi e c}{\alpha^{3} \lambda^{2}}\right)^{2}=2.198300502 \mathrm{E} 16 \mathrm{j} / \mathrm{m}^{3}
$$

The equation defining the volume within which such high energy densities make sense is derived in reference [14] and is also shown further on as Equation (50).

\section{The Internal Electromagnetic Structure of the Electron Carrying Energy}

It has been established long ago that the electron is an electromagnetic particle. However, the nature of its carrying energy could never be clarified until Marmet derived Equation (2) from the Biot-Savart equation, leading to Equation (13), that reveals that this carrying energy is made of 2 parts, that is, one half sustaining the momentum $\Delta K$ of the particle, and the other half identified by Marmet as an amount of magnetic energy that adds a relativistic mass increment $\Delta m_{m}$ to the invariant rest mass of the particle in motion.

Since the electric charge of the electron has been systematically proven over the course of the past century to remain invariant, irrespective of its velocity, the related intrinsic electric $\boldsymbol{E}$ field that was established with the second Equation (22) can be expected to also remain invariant, and to remain Maxwell equations compliant, so does its intrinsic magnetic $\boldsymbol{B}$ field established with the first Equation (22).

As put in perspective with Equation (13), since the $\Delta m_{m}$ magnetic mass increment identified by Marmet increases in the exact same proportion as the $\Delta K$ momentum energy of the electron, and that these two energy amounts can not be part of the energy quantum making up the invariant rest mass of the electron, this gives us a first conclusive clue that this carrying energy also is electromagnetic in nature, since its magnetic field cannot be dissociated from electromagnetism and consequently from Maxwell's equations.

This total amount of carrying energy represented by Equation (13) can thus be logically represented with the following relational equation: 


$$
E_{\text {(Total electron carrying energy) }}=E_{\text {(Momentumenergy) }}+E_{\text {(Magnetic mass increment energy) }}
$$

But to remain consistent with electromagnetism, it seems impossible that this magnetic energy component would not be involved in some cyclic process of electromagnetic oscillation between this magnetic state and a yet to be identified "electric" state, that could potentially be represented as a reciprocating oscillation between both states, in conformity with the very foundation of Maxwell's theory, to the effect that for electromagnetic energy to even exist, both of these aspects must mutually induce each other [20]:

$$
\begin{aligned}
& E_{(\text {Total carrying energy })} \\
& =E_{(\text {Momentum energy })}+\left[E_{(\text {Electric state })} \cos ^{2}(\omega t)+E_{(\text {Magnetic state })} \sin ^{2}(\omega t)\right]
\end{aligned}
$$

It is at this point that a huge leap "out of the box" needs to be made, as the saying goes, because since this magnetic mass increment freshly identified by Marmet has been proven to physically exist by transverse interaction with relativistically moving electrons in experiments carried out by Walter Kaufman at the beginning of the 20th century [21], this means that the energy making up this "mass increment" can only physically exist just like the energy making up the invariant rest mass of the electron. And finally so must it also be for its momentum energy, despite the long held conclusion that it exists only inasmuch as its velocity can be expressed.

This conclusion leads to converting relational Equation (32) to the following electromagnetic form, representing this electromagnetic oscillation as a simple harmonic LC reciprocal transverse oscillation-in conformity with the fact that the $\boldsymbol{E}$ and $\boldsymbol{B}$ fields must be perpendicular to the direction of motion-between an electric state and a magnetic state of the energy making up the magnetic mass increment identified by Marmet:

$$
E_{(\text {Total carrying energy })}=\frac{h c}{2 \lambda}+\left[\frac{e^{2}}{2 C_{\lambda}} \cos ^{2}(\omega t)+\frac{L_{\lambda} i_{\lambda}^{2}}{2} \sin ^{2}(\omega t)\right]
$$

where

$$
E_{E(\max )}=\frac{q^{2}}{2 C} \text { and } E_{B(\max )}=\frac{L i^{2}}{2}
$$

The definitions of the subcomponents $\mathrm{C}, \mathrm{L}$ and $\mathrm{i}$ are provided further on with Equations (45) and (47).

In this transitory form, Equation (33) may give the impression that the electromagnetic energy of the $\Delta m_{m}$ half-quantum oscillates "longitudinally", so to speak, moving in the same vectorial direction as its $\Delta K=h c / 2 \lambda$ momentum energy, but we will see further on that it can oscillate only transversally in conformity with Maxwell's equations, when the vectorial infrastructure will be put in place with Equation (48).

We will also see further on that the oscillation of the magnetic energy of this magnetic mass increment, between a state of maximum presence and one of zero presence as a function of its electromagnetic frequency is key to understanding 
the various resonance states of the electron, that is its Zitterbewegung on one hand, and also its axial resonance state when captive into a least action electromagnetic equilibrium state in an authorized atomic orbital.

Indeed, it can be established, as we will see further on, that even the magnetic energy of the invariant rest mass of the electron can only be involved separately in a similar harmonic oscillating motion between maximum presence and zero presence in space [17], and that the same oscillation characterizes the magnetic energy of the two types of elementary components making up all nucleons and of their respective carrying energies, that is, the up quark and the down quark [18].

\section{Correlating Classical Mechanics and Relativistic Mechanics via Electromagnetism}

The first benefit of representing the electron carrying energy with LC Equation (33), is the ease with which it allows visualizing its electromagnetically oscillating half as oscillating perpendicularly to the direction of motion of the energy sustaining its translational momentum $(\Delta K=h c / 2 \lambda)$, which clearly matches, as previously mentioned, the well known perpendicular relation between the $E$ and $\boldsymbol{B}$ fields of Maxwell's theory with respect to the direction of motion of any point on the wave front of his theoretical continuous electromagnetic wave in spherical expansion from its point of emission.

In turn, this clear separation between the unidirectionally oriented momentum energy and the transversally oscillating energy of the carrying energy quantum allowed directly upgrading Newton's non-relativistic kinetic energy equation $K=m v^{2} / 2$ to a fully relativistic electromagnetic form [22]:

$$
\frac{v^{2}}{c^{2}}=\frac{4 \lambda \lambda_{C}+\lambda_{C}^{2}}{\left(2 \lambda+\lambda_{C}\right)^{2}}
$$

One unexpected outcome of the establishment of Equation (35) was that when using the wavelength of the carrying energy induced at mean ground state orbital distance from the nucleus of the hydrogen atom $(4.556335261 \mathrm{E}-08 \mathrm{~m})$, it directly provides the fine structure constant $\alpha[23]$ :

$$
\alpha=\frac{v}{c}=\frac{\sqrt{\lambda_{C}\left(4 \lambda+\lambda_{C}\right)}}{\left(2 \lambda+\lambda_{C}\right)}=7.297352533 \mathrm{E}-03
$$

More surprising yet! Further dividing Equation (36) by $2 \pi$ provides the exact fine structure constant $\alpha$ related electron " $g$ " factor discovered by Julian Schwinger in 1948 [23] [24]:

$$
\begin{aligned}
& \text { Electron magnetic momen magnetic drift } \\
& =\frac{\sqrt{\lambda_{C}\left(4 \lambda+\lambda_{C}\right)}}{2 \pi\left(2 \lambda+\lambda_{C}\right)}=\frac{\delta \mu}{\mu_{B}}=\frac{\alpha}{2}=1.161386535 \mathrm{E}-3
\end{aligned}
$$

The fact that electromagnetic Equation (35) is relativistic by structure, also allows deriving the 4 standard relativistic equations. First in line is the relativistic 
momentum energy equation, now amended to also account for the presence of the magnetic mass increment $\Delta m_{m}$ of elementary particles carrying energy [22]:

$$
K=2 m_{0} c^{2}(\gamma-1)
$$

For the first time ever also, apparently, the Lorentz gamma factor equation was derived directly from an electromagnetic equation in reference [22], that is, from Equation (35), instead of from strictly mathematical geometric and trigonometric methods, as has been systematically done since Woldemar Voigt came up with the idea in 1887 [11] [22] [23] [25] [26]:

$$
\gamma=\frac{1}{\sqrt{1-v^{2} / c^{2}}}
$$

The third relativistic equation derived was of course the relativistic mass equation of a moving elementary particle in motion [22]:

$$
E=\gamma m c^{2} \text { where } \gamma m=m_{0}+\Delta m_{m}
$$

And finally, the relativistic energy-momentum relation equation [27]:

$$
E^{2}=(p c)^{2}+\left(m c^{2}\right)^{2}
$$

Thus conclusively demonstrating that classical relativistic equations and electromagnetic equations can reversibly be derived from each other.

Besides Equation (35) making use of the wavelengths defined with Equations (14) and (15) from which all classical relativistic equations can be derived, a second and even more fundamental electromagnetic equation was derived from upgrading Newton's kinetic energy equation to full electromagnetic status [22]. It is the following equation that directly makes use of the "quantities of energy" separately making up the invariant rest mass of the electron, its momentum, and finally its magnetic mass increment, the last two constituting its carrying energy. It is the following form:

$$
\frac{\left(h c / \lambda+2 h c / \lambda_{C}\right)^{2}-\left(2 h c / \lambda_{C}\right)^{2}}{\left(2 L_{C} i_{C}^{2}+L_{\lambda} i_{\lambda}^{2}\right)^{2}}=\frac{v^{2}}{c^{2}}
$$

that resolves to

$$
v=c \frac{\sqrt{4 E K_{\text {momentum }}+\left(K_{\text {momentum }}\right)^{2}}}{2 E+K_{\text {magnetic }}}
$$

where $E$ represents the energy of the invariant rest mass of the electron, $K_{\text {momentum }}$ is the $\Delta K$ momentum energy provided by the carrying energy, and $K_{\text {magnetic }}$ is the energy going into the $\Delta m_{m}$ magnetic mass increment provided by the carrying energy of the electron.

What is so fundamental and important about this equation, is that when the energy of the electron rest mass is reduced to zero, leaving only its carrying energy in the equation, we end up with an equation that systematically provides the speed of light in an invariant manner, whatever the sum of the two 
half-quanta always equal by structure of the momentum energy and of the energy of the remaining magnetic mass; a velocity possible only for free moving electromagnetic energy:

$$
v=c \frac{K_{\text {momentum }}}{K_{\text {electromagnetic }}}=\frac{\Delta K}{\Delta m_{m} c^{2}}=c \frac{h c / 2 \lambda}{L_{\lambda} i_{\lambda}^{2}}=c \frac{1}{1}=299792458 \mathrm{~m} / \mathrm{s}
$$

where

$$
L=\frac{\mu_{0} \alpha \lambda}{8 \pi^{2}} \text { and } i=\frac{2 \pi e c}{\alpha \lambda}
$$

Since Marmet's contribution allows conclusively establishing that $\Delta m_{m}$ from Equation (2) and $\Delta K$ from Equation (6) will systematically be equal whatever total amount the sum of their energies will be, these two energy values will systematically simplify to 1 in Equation (44) whatever energy amount of electromagnetic energy is represented by its wavelength $\lambda$.

This means that for the first time, we have a conclusive clue regarding the possible internal electromagnetic structure of localized free moving electromagnetic photons, that is, electromagnetic photons that would not be slowed down by having to "carry and propel", so to speak, the translationally inert electromagnetic mass of an electron on top of having to carry and propel their own electromagnetic mass complement. So LC Equation (33) could consequently be applied as well to free moving electromagnetic photons and to the electron's carrying energy, which would fully justify naming the latter a "carrier-photon".

\section{The de Broglie Double-Particle Electromagnetic Photon}

So let us now consequently re-identify Equation (33) as describing the total energy of a free-moving electromagnetic photon and analyze further its structure:

$$
E_{\text {(Total photon energy) }}=\frac{h c}{2 \lambda}+\left[\frac{e^{2}}{2 C_{\lambda}} \cos ^{2}(\omega t)+\frac{L_{\lambda} i_{\lambda}^{2}}{2} \sin ^{2}(\omega t)\right]
$$

Of course, the $L$ and $i$ variables definitions of Equations (45) still apply and the definition of $C$ established in reference [8] is:

$$
C=2 \varepsilon_{0} \alpha \lambda
$$

We first observe that the electric phase of the electromagnetic transverse oscillation between magnetic and electric states seems to involve a pair of charges, which is a possibility that has been a major stumbling block in electromagnetic theory ever since Maxwell established his theory of light propagation on the then axiomatic concept that the very existence of this energy mandated that both $E$ and $\boldsymbol{B}$ fields mutually induce each other for the energy to even exist.

Even if the resulting theory has proven out of any doubt its absolute conformity with experience at the macroscopic level, the origin of the "displacement current" that would involve such a local motion of some postulated double electric charges to induce the magnetic field, while they supposedly close in on each 
other, inducing the magnetic field, to then be re-induced themselves as the magnetic field regresses, could never be clarified either experimentally nor theoretically.

In a search to identify these still hypothetical charges at the submicroscopic level, de Broglie tried in the 1930's to establish a clear internal electromagnetic mechanics of the localized photon grounded on the characteristics of the wave function.

It turns out that he did correctly establish that such a permanently localized photon should satisfy the Bose-Einstein's statistic and Planck's Law, explain the photoelectric effect while obeying Maxwell's equations and remain in accordance with the properties of Dirac's theory of complementary corpuscles symmetry, if it involves two half-photons of spin $1 / 2$,

“...that must be complementary with respect to each other in the same manner that the positive electron (the positron) is complementary to the negative electron in the Dirac Hole Theory... Such a complementary couple of particles is likely to annihilate at the contact of matter by relinquishing all of its energy, which perfectly accounts for the characteristics of the photoelectric effect... The photon, being made up of two elementary particles of spin $h / 4 \pi$, will obey the Bose-Einstein statistic as required by the precision of Planck's law for the black body.... this model of the photon allows the definition of an electromagnetic field linked to the probability of annihilation of the photon, a field that obeys Maxwell's equations and has all the characteristics of electromagnetic light waves." ([28], p. 277).

His attempts to define the localized electromagnetic photon from the properties of the wave function were unsuccessful to the point that he finally concluded in 1936 that it was impossible to exactly represent elementary particles in the frame of $4 \mathrm{D}$ spacetime geometry, in his view too restrictive, hinting that if this frame could eventually be escaped from, such a description could become possible:

"...the non-individuality of particles, the exclusion principle and exchange energy are three intimately related enigmas, all three are tied to the impossibility of exactly representing elementary physical entities within the frame of continuous three dimensional space (or more generally of continuous four dimensional space-time). Some day maybe, by escaping from this frame, will we better grasp the meaning, still quite cryptic today, of these major guiding principles of the new physics." ([28], p. 273).

Retrospectively, it seems that in the restricted frame of $4 \mathrm{D}$ space geometry, reverse engineering the description of the electromagnetic photon from the characteristics of wave function that was not initially grounded on electromagnetism to start with, was an impossible task, since, let's remember, it was introduced by Schrödinger to represent a state of resonance in the classical resonance mechanics sense, stemming from de Broglie's comparison with well known classical mechanics resonance states [1]. See also Equation (1). We will come back further on to this issue of reverse engineering. 
The only real relation that can exist between Schrödinger's wave function and the "electromagnetic" resonance state of the electron captive in least action electromagnetic resonance state in the ground state orbital of the hydrogen atom can then only be a description of the spatial resonance volume within which all of the electron energy is expected to be contained, and gives no clue whatsoever as to the nature of the "electromagnetic resonator" whose resonance characteristics could explain this resonance volume.

Besides, the very idea that half the energy of the quantum could behave as 2 half-quantities displaying "electric" properties as they close in on each other while at the same time concentrically accumulating as a single quantity within the same volume of space that would display "magnetic" properties goes directly against logic when considering that this energy would be a "physically existing substance" as the previous analysis leads to conclude.

This mechanical impossibility that comes to light when attempting to represent in the same volume of space the mutual induction of the electric and magnetic aspects of localized electromagnetic quanta by reciprocating swing, effectively correlates with de Broglie's conclusion that elementary particles cannot be represented in the too restrictive frame of $4 \mathrm{D}$ spacetime geometry.

\section{Expanding the Space Geometry}

In Maxwell's wave theory, it is well understood that the continuous wave concept imposes that both $\boldsymbol{E}$ and $\boldsymbol{B}$ fields of Maxwell's theory must be "in phase" for the wave to exist and propagate. But contrariwise, the idea that the energy of localized electromagnetic quanta could exist, due to a self-sustaining reciprocating LC oscillation, imposes that both fields be $180^{\circ}$ "out of phase" for such an LC oscillation to be mechanically possible.

A close examination of the traditional graphic representations of Maxwell's theory electromagnetic phases and of his equations reveals however that both in phase alignment and $180^{\circ}$ out of phase alignment result in the very same configuration (Figure 2).

This reveals that although $180^{\circ}$ out of phase alignment is incompatible with Maxwell's continuous electromagnetic wave theory, it is perfectly allowed by his equations, and that true $180^{\circ}$ out of phase alignment, involving that electric energy reaching minimum while the magnetic energy reaches maximum and the reverse is indeed allowed and is effectively consistent with representing a self-sustaining electromagnetic quantum by a reciprocating LC oscillating process (Figure 3). Moreover, it is consistent with the very foundation of Maxwell's theory to the effect that both fields have to mutually induce each other for the energy to even exist.

With regard to the mechanical impossibility that 2 half-quantities of a physically existing "substance" displaying "electric" properties as they close in on each other while at the same time concentrically accumulating in a single quantity that would display "magnetic" properties within the same volume of space, it is this very mechanical impossibility that gave rise to the idea that the solution 


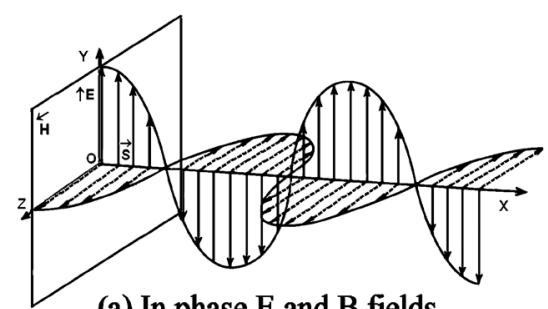

(a) In phase $\mathrm{E}$ and $\mathrm{B}$ fields

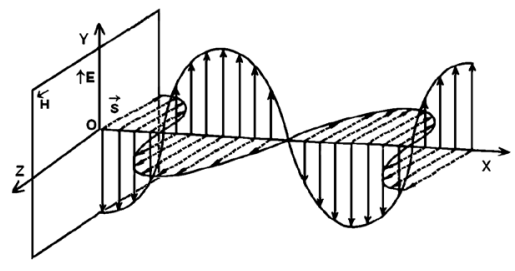

(b) $180^{\circ}$ out of phase fields

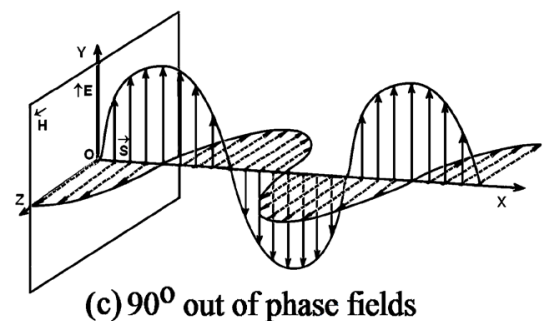

Figure 2. Traditional representations of electromagnetic fields in phase, $180^{\circ}$ out of phase, and $90^{\circ}$ out of phase, in classical electromagnetism.

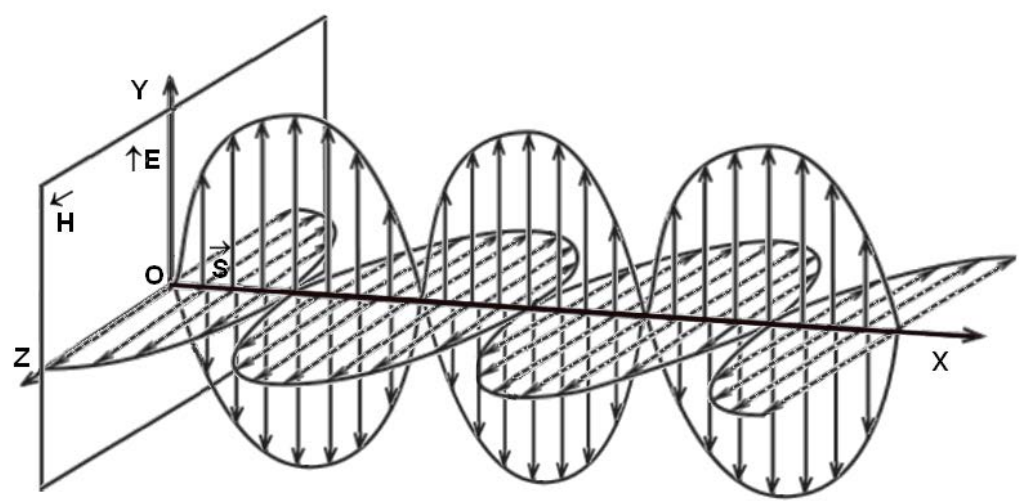

Figure 3. $180^{\circ}$ out of phase representation of E and B fields of Maxwell's theory for an LC oscillation.

could be for the magnetic quantity to "grow", so to speak, into a different space while both charges were closing in within the first space, and inversely.

And without even going so far as to assume the real physical existence of such a second space, it so happens that from the vectorial perspective, it is rather easy to represent such a multi-spaces complex, and it is particularly easy to represent vectorially both $\boldsymbol{E}$ and $\boldsymbol{B}$ fields of the $\Delta m_{m}$ magnetic mass half-quantum as transversally oscillating with respect to the direction of motion of the $\Delta \mathrm{K}$ momentum half-quantum, in conformity with Maxwell's equations.

In this particular case, it so happens that the well known vectorial cross product of the magnetic $\boldsymbol{B}$ field vector and electric $\boldsymbol{E}$ field vector, both perpendicular to each other, resolving in a third vector perpendicular to the first two and representing the phase velocity (Figure $4(a)$ ), which is the triple orthogonal relation that maps the direction of motion at the speed of light of any point of the wavefront of Maxwell's hypothetical spherically expanding continuous electromagnetic wave, gives us a solid footing to explore such a possibility. 


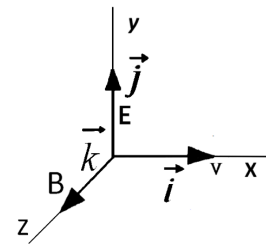

(a)

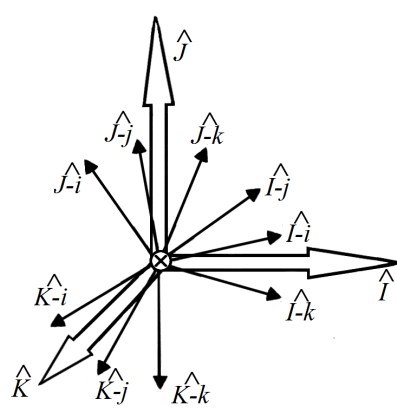

(b)

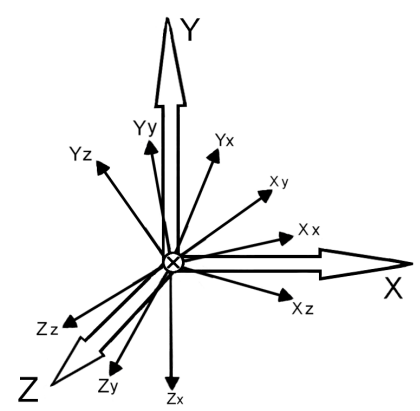

(c)

Figure 4. Major and minor unit vectors set applicable to the trispatial geometry.

The method consists in geometrically "exploding", so to speak, each of the 3 standard electromagnetic vectors $\boldsymbol{i}, \boldsymbol{j}$ and $\boldsymbol{k}$, applicable to normal space into 3 full blown 3D vectorial spaces of their own (Figure 4(b)), each of the three X, Y and $\mathrm{Z}$ spaces (Figure 4(c)) remaining perpendicular to the other two and all of them remaining connected via their common origin, previously identified as being midpoint $\otimes$ between a pair of charges in Figure 1, and that can now be seen as a transit point for the energy, that would be located at the center of each electromagnetic elementary quantum, and through which the "substance" of the energy quantum would be free to travel as if between communicating vessels, according to their required electromagnetic reciprocating motion, without the illogical interpenetration of the energy substance that would prevent such a reciprocating motion within the more limited frame of a single 3D space geometry.

Contrary to expectation, it turns out to be relatively easy to mentally visualize such a trispatial mutually orthogonal 9-dimensional geometric complex. It suffices to imagine each of the 3 sets of minor vectors $i, j$ and $k$ of Figure 4(b) as if they were folded metaphoric 3-rib umbrellas.

This allows us to mentally open at will any one of them, one at a time, to full 3-axes orthogonal extension to observe the behavior of the energy quantum substance in this fully deployed 3D space during each phase of the oscillating motion. Figure 4(b) and Figure 4(c) show the dimensions of the 3 spaces half deployed to allow clear unique identification of each of the 9 resulting orthogonal inner axes, which allows simple mathematical and vectorial identification of the inner motion of the energy within each space without changing nor invalidating in any way any of the traditional vectorial representations applied in the normal 4D space geometry to represent electromagnetic energy, in the traditional mechanics.

In this space geometry, the momentum energy that translationally propels elementary particles is unidirectional by definition, and is set by structure to be insensitive to any transverse interaction, which directly correlates with the observations made by Walter Kaufman between the longitudinal inertia and the transverse inertia of electrons moving at relativistic velocities in a bubble chamber [21], when he observed that although both half-quanta $\Delta K$ and $\Delta m_{m}$ could be measured longitudinally in addition to the electron rest mass, only the $\Delta m_{m}$ 
half-quantum could be measured transversally in addition to the electron rest mass.

The same property will cause the pair of opposite signs "electric charges" of an electromagnetic quantum unidirectionally moving toward or away from each other on the $Y-y / Y-z$ plane within Y-space to appear to be neutral with respect to the orthogonally oriented $\mathrm{Y}$ - $\mathrm{x}$ axis and to not even be detectable as perceived from X-space, which is the space from which we observe objective reality, which correlates with the observed fact that electromagnetic photons do not seem to possess electric charges [8] [15], despite the physical incompatibility of such an absence with Maxwell's theory.

The same indetectability and apparent absence of opposite signs charges will characterize the pairs of "neutrinic charges" unidirectionally moving toward or away from each other on the X-y/X-z plane within X-space [16] [17].

The fact that the pair of "electric charges" can only move in opposite directions on the $\mathrm{Y}-\mathrm{y} / \mathrm{Y}-\mathrm{z}$ plane is what explains why photons can be polarized at any angle perpendicularly to their direction of motion along the $\mathrm{X}-\mathrm{x}$ axis of normal $\mathrm{X}$-space. Obviously, the same polarizability property will apply to the pair of "neutrinic charges" moving in opposite directions on the X-y/X-z plane.

Finally, any amount of energy now oscillating between $\mathrm{Y}$ and $\mathrm{Z}$ spaces finds itself oscillating transversally "by structure" with respect to normal X-space, and will thus appear to possess omnidirectional inertia as perceived from $\mathrm{X}$-space, that is, will behave as if it was "massive" in the sense understood in classic$\mathrm{al} /$ relativistic mechanics, as perceived from the $\mathrm{X}$-space.

This expanded space geometry was first proposed at the Congress-2000 event held at the St Petersburg State University in July of 2000 [29]. It is introduced and put in perspective in reference [12] with respect to the traditional multidimensional geometries conceived of in previous historical attempts at resolving the remaining issues of fundamental physics, and is completely described in Reference [8].

\section{Fundamental Symmetry Maintained by Structure}

One aspect of utmost interest of the trispatial geometry is that the fundamental principle of symmetry is respected by structure for all aspects of the distribution of the energy of an electromagnetic quantum.

The energy is systematically distributed between one half remaining unidirectional in one of the spaces while the other half cyclically oscillates in perpendicular orientation with respect to the first half by structure (half-half symmetry), which is what immediately reveals that in this space geometry, the speed of light can only be an invariant equilibrium velocity in vacuum in all cases of free moving electromagnetic photons, due to this half-half energy distribution mandated by structure between both half-quanta [8].

Within electrostatic Y-space where both electric charges-for free moving photons and carrier-photons-axially oscillate toward and away from each other [8] [15], and within normal X-space where both neutrinic charges (for massive 
electron, positron, up quark and down quark-speaking only of the stable states) [16] [17] [18] also axially oscillate toward and away from each other in the same manner on the plane perpendicular to the space where their unidirectional complement resides, always symmetrically possessing equal amounts of energy and opposite directions, along which the varying distance between them, provide the corresponding varying intensity of the opposite signs of their charges (symmetry between the energy amounts and also between the intensities of the opposite signs of their charges within $\mathrm{Y}$-space and $\mathrm{X}$-space).

Within magnetostatic Z-space where a single quantity of the energy grows to a maximum while leaving Y-space for photons and carrier-photons [8] [15], or while leaving X-space for massive particles [16] [17] [18], this single quantity, after having reached maximum volume presence in $\mathrm{Z}$-space, regresses toward zero presence in this space while the energy crosses over back into the $\mathrm{Y}$-space-or $\mathrm{X}$-space-it was in previously (symmetry between the increasing phase and the decreasing phase of the energy presence within magnetostatic Z-space).

In normal X-space, neutrino energy can be released only as identical pairs in opposite directions perpendicularly to the direction of motion of the unidirectional energy present in this space belonging to a newly created massive elementary particle-electron, muon or tau-that sheds in this manner an excess metastable initial excess amount of mass [16] (More on this issue later on).

And global symmetry is also preserved since the time-varying space-wise moving electric dipole is permanently counterbalanced by a related time-varying growing and decreasing magnetic dipole oriented perpendicularly, with both dipoles remaining perpendicular to the direction of motion of the photon in space, thus obeying the triple orthogonality required for plane wave treatment in Maxwell's theory's for straight line motion of electromagnetic energy [8].

\section{The Trispatial Photon Equation}

The first inner electromagnetic structure that the trispatial geometry allowed defining was that of the localized photon that de Broglie concluded could not be defined within the too restrictive confines of $3 \mathrm{D}$ space [8], and that graphically shows with Figure 5 the transverse harmonic oscillation sequence of the energy of the photon as represented with Equation (46).

Figure 5 indeed allows visually representing the complete time varying sequence of the transverse oscillation of the energy of the electromagnetic half-quantum within the trispatial complex. Figure 5(a) shows both inner opposite charges, measurable as generating the $\mathrm{E}$ field of the photon at its maximum value, having reached maximum transverse distance within electrostatic $\mathrm{Y}$-space, followed by Figure 5(b) showing the energy of both charges transferring to magnetostatic Z-space.

Then comes Figure 5(c) showing the energy of both charges having completely entered Z-space in omnidirectional expansion, now measurable as generating the $B$ field of the photon at its maximum value, followed by Figure 5(d) showing the energy of the single magnetic component transferring back into 


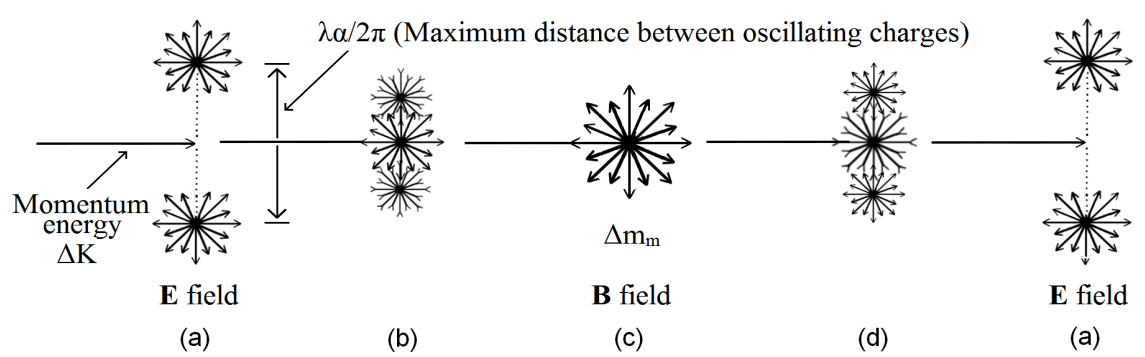

Figure 5. Complete time varying cycle of the energy in transverse oscillation of the electromagnetic half-quantum of the double-particle photon while its momentum sustaining unidirectional half-quantum propels it translationally.

electrostatic Y-space. Finally, the final Figure 5(a) shows all of the magnetic energy having transferred back into $\mathrm{Y}$-space as two opposite charges having reached again maximum transverse distance, and again measurable as the $\mathrm{E}$ field of the photon, ready for the next cycle to begin.

As mentioned previously, the double-particle photon concept is an original idea of Louis de Broglie, and the complete analysis of its elaboration in the trispatial geometry is available in reference [8], where the complete development of its trispatial LC equation is elaborated from the inductance and capacitance representations of electromagnetic energy:

$$
E \vec{I} \vec{i}=\left(\frac{h c}{2 \lambda}\right)_{X} \vec{I} \vec{i}+\left[2\left(\frac{e^{2}}{4 C}\right)_{Y}(\vec{J} \vec{j}, \vec{J} \overleftrightarrow{j}) \cos ^{2}(\omega t)+\left(\frac{L i^{2}}{2}\right)_{Z} \stackrel{\leftrightarrow}{K} \sin ^{2}(\omega t)\right]
$$

and also of the same LC formulation making use of the more familiar $\boldsymbol{E}$ and $\boldsymbol{B}$ fields defined with Equations (23):

$$
E \vec{I} \vec{i}=\left(\frac{h c}{2 \lambda}\right)_{X} \vec{I} \vec{i}+\left[2\left(\frac{\varepsilon_{0} E^{2}}{4}\right)_{Y}(\vec{J} \vec{j}, \vec{J} \overleftrightarrow{j}) \cos ^{2}(\omega t)+\left(\frac{\boldsymbol{B}^{2}}{2 \mu_{0}}\right)_{Z} \stackrel{\leftrightarrow}{K} \sin ^{2}(\omega t)\right] V
$$

Where volume $V$ is the theoretical stationary isotropic volume that the incompressible oscillating kinetic energy of the photon would occupy if it was immobilized as a sphere of isotropic density, as derived in reference [14]:

$$
V=\alpha^{5} \frac{\lambda^{3}}{2 \pi^{2}}
$$

\section{The Trispatial Electron Equation}

It is well established that electromagnetic photons of $1.022 \mathrm{MeV}$ or more can be destabilized into converting to an electron-positron pair [17]. However, it so happens that all of the energy making up the two $0.511 \mathrm{MeV} / \mathrm{c}^{2}$ rest masses of both electron and positron is electromagnetic in nature and thus reside within $\mathrm{Y}$-space and Z-space in the new trispatial geometry, while the half-quantum of the whole initial quantum of a $1.022 \mathrm{MeV}$ photon that resides in $\mathrm{X}$-space before decoupling is vectorially unidirectional by definition. This means that Nature has found some way to force this $\Delta K$ unidirectional momentum energy to re-orient transversally for it to become part of the electromagnetic mass of both emergent massive particles. 
One of the most interesting features of the trispatial geometry is that it effectively allows establishing a clear mechanical process by which this $\Delta \mathrm{K}$ unidirectional energy of the half-quantum sustaining the momentum of a moving electromagnetic photon of $1.022 \mathrm{MeV}$ can cross over into the orthogonal electrostatic Y-space and magnetostatic Z-space during the decoupling process, and so acquire the transverse orientation property that characterizes the complete mass energy of both electron and positron of the pair resulting from the separation process in the trispatial geometry [17].

By the same token, the very mechanics of transfer of this momentum energy into Y-space to end up defining the invariant unit charge of both electron and positron, also forces by structure the other half of the energy of each particle of the pair in process of separation to now start oscillating between Z-space and $\mathrm{X}$-space for the symmetric energy distribution be maintained in the trispatial complex, resulting in the establishment of a double component state within $\mathrm{X}$-space separating in a manner identical to the behavior of the pair of "electric charges" of the photon within Y-space, that are traditionally represented by $e^{2}$, but that now require to be identified with a new denomination since they cannot have the "electric" characteristic anymore, that belongs by definition only to energy present in $\mathrm{Y}$-space, in this trispatial complex. Pending clear identification, the first-draft symbol that best fitted them was then $(e)^{2}$.

As we will see further on, deeper analysis succeeded in relating these double $(e)^{2}$ "non-electric charges" to the emission of neutrinos, which earned them the name of "neutrinic charges" in the subsequent descriptions [16] [17].

The following trispatial LC equations were then defined to describe the corresponding inner trispatial energy structure of the mass of the electron and positron:

$$
\begin{aligned}
& E \overrightarrow{\mathbf{0}}=m_{e} c^{2} \overrightarrow{\mathbf{0}} \\
& =\left[\frac{H}{2 \lambda_{C}}\right]_{Y} \overrightarrow{\boldsymbol{J}} \overrightarrow{\boldsymbol{i}}+\left(2\left[\frac{\left(e^{\prime}\right)^{2}}{4 C_{C}}\right]_{X}\left(\overrightarrow{\boldsymbol{I}} \overrightarrow{\boldsymbol{j}}, \overrightarrow{\boldsymbol{I}} \stackrel{\boldsymbol{j}}{)} \cos ^{2}(\omega t)+\left[\frac{L_{C} i_{C}^{2}}{2}\right]_{Z} \stackrel{\leftrightarrow}{\boldsymbol{K}} \sin ^{2}(\omega t)\right)\right.
\end{aligned}
$$

and

$$
\begin{aligned}
& E \overrightarrow{\mathbf{0}}=m_{e} c^{2} \overrightarrow{\mathbf{0}} \\
& =\left[\frac{H}{2 \lambda_{C}}\right]_{Y} \overrightarrow{\boldsymbol{J}} \overleftarrow{\boldsymbol{i}}+\left(2\left[\frac{\left(e^{\prime}\right)^{2}}{4 C_{C}}\right]_{X}(\overrightarrow{\boldsymbol{I}} \overrightarrow{\boldsymbol{j}}, \overrightarrow{\boldsymbol{I}} \overleftarrow{\boldsymbol{j}}) \cos ^{2}(\omega t)+\left[\frac{L_{C} i_{C}^{2}}{2}\right]_{Z} \stackrel{\leftrightarrow}{\boldsymbol{K}} \sin ^{2}(\omega t)\right)
\end{aligned}
$$

Subsequent reformulation of the same LC equations making use of the more familiar $E$ and $B$ fields defined with Equations (23) then forced the identification of the component pair $(e)^{2}$ as "neutrinic charges" $\left(v^{2}\right)$ in references [16] [17] for reasons that will soon become obvious:

$$
m_{0} \overrightarrow{\mathbf{0}}=\frac{V_{m}}{c^{2}}\left\{\left[\frac{\varepsilon_{0} \boldsymbol{E}^{2}}{2}\right]_{Y} \overrightarrow{\boldsymbol{J}} \overrightarrow{\boldsymbol{i}}+\left[2\left(\frac{\varepsilon_{0} v^{2}}{4}\right)_{X}(\overrightarrow{\boldsymbol{I}} \overrightarrow{\boldsymbol{j}}, \overrightarrow{\boldsymbol{I}} \overleftrightarrow{\mathbf{j}}) \cos ^{2}(\omega t)+\left(\frac{\boldsymbol{B}^{2}}{2 \mu_{0}}\right)_{Z} \stackrel{\leftrightarrow}{\boldsymbol{K}} \sin ^{2}(\omega t)\right]\right\}
$$


where $v$ (Greek letter nu) represent the "neutrinic" field equation [16] [17] that now represents the double "neutrinic charges" whose energy calculation is identical to that of the electric $E$ field equation, but that now oscillate in opposite directions on the $\mathrm{X}-\mathrm{y} / \mathrm{X}-\mathrm{z}$ plane of X-space, within the trispatial energy structure of elementary massive particle masses in Equations (51) and (52), just like the "electric charges" oscillate in opposite directions on the $\mathrm{Y}-\mathrm{y} / \mathrm{Y}-\mathrm{z}$ plane of Y-space, within the photon or carrier-photon trispatial energy structure [8] [15] in Equation (48) and (49). Here are the definitions of the required isotropic volume and of the neutrinic field:

$$
V_{m}=\frac{\alpha^{5} \lambda_{C}^{3}}{2 \pi^{2}} \text { and } v=\frac{\pi\left(e^{\prime}\right)}{\varepsilon_{0} \alpha^{3} \lambda_{C}^{2}}
$$

where $(e)^{2}$ is assigned the same numerical value grounded on the value of the unit electric charge $e=1.602176462 \mathrm{E}-19$, since a pair of such components represents by structure the same maximum amount of energy in the trispatial electron structure, that is, half the rest mass of the electron when reaching maximum distance from each others within $\mathrm{X}$-space when it splits in two equal quantities.

Modelled on Equation (49) for the free moving photon, the equation for the electron carrier-photon making use of $\mathbf{E}$ and $\mathbf{B}$ fields can now be formulated as follows, providing the same energy as the corrected classical relativistic kinetic Equation (13):

$$
E_{K} \overrightarrow{\boldsymbol{I}} \overrightarrow{\boldsymbol{i}}=\left[\frac{h c}{2 \lambda}\right]_{X} \overrightarrow{\boldsymbol{I}} \overrightarrow{\boldsymbol{i}}+\left[2\left(\frac{\varepsilon_{0} \boldsymbol{E}_{K}^{2}}{4}\right)_{Y}(\overrightarrow{\boldsymbol{I}} \overrightarrow{\boldsymbol{j}}, \overrightarrow{\boldsymbol{I}} \overleftrightarrow{\boldsymbol{j}}) \cos ^{2}(\omega t)+\left(\frac{\boldsymbol{B}_{K}^{2}}{2 \mu_{0}}\right)_{Z} \stackrel{\leftrightarrow}{\boldsymbol{K}} \sin ^{2}(\omega t)\right] V_{K}
$$

which now allows representing the combined fields equations of the electron and its carrying energy as in Table 1.

In fact, the carrier-photon provides the electron with the ambient $\boldsymbol{E}$ and $\boldsymbol{B}$ fields that permanently determine its velocity and direction of motion, when they can be expressed, in accordance with the Lorentz equation $\boldsymbol{F}=q(\boldsymbol{E}+\boldsymbol{v} \times \boldsymbol{B})$ previously mentioned. More precisely, it constantly obeys the triple orthogonal relation $\boldsymbol{V}=$ $\boldsymbol{E} / \boldsymbol{B}$ stemming from the Lorentz equation imposed to it by the $\boldsymbol{E}$ and $\boldsymbol{B}$ fields of its carrier-photon, whose intensity determines its velocity, and whose relative densities equilibrium determines its trajectory, default equal densities of both $E$ and $\boldsymbol{B}$ fields resulting in straight line motion of the electron [14].

In turn, the $\boldsymbol{B}$ field of the electron carrier-photon constantly tends to align its relative magnetic polarity orientation, that is, its relative spin orientation, in least action antiparallel alignment with respect to the $\boldsymbol{B}$ field of the rest mass energy of the electron that it carries, and the combined resultant of which constantly tends to align in a least action antiparallel orientation with respect to the resultant of the $\boldsymbol{B}$ fields of the surrounding particles, thus with respect to the ambient macroscopic $\boldsymbol{B}$ field resulting from the addition of the surrounding $\boldsymbol{B}$ fields. 
Table 1. Combined fields equations of the moving electron and its carrier-photon.

\begin{tabular}{|c|c|c|}
\hline & $\begin{array}{c}\text { Momentum } \\
\text { kinetic } \\
\text { energy } \\
\text { in X-Space } \\
\text { (normal space) }\end{array}$ & $\begin{array}{l}\text { Energy located in } \mathrm{Y} \text { and } \mathrm{Z} \text { spaces } \\
\text { making up } \\
\text { the translationally inert mass } \\
\text { of the particle in motion }\end{array}$ \\
\hline $\begin{array}{l}\text { Rest mass } \\
\text { energy } \\
\left(m_{0} c^{2}\right)\end{array}$ & & $\left\{\left(\frac{\varepsilon_{0} \boldsymbol{E}^{2}}{2}\right)_{Y} \overrightarrow{\boldsymbol{J}} \overrightarrow{\boldsymbol{i}}+\left(\left(\frac{\boldsymbol{B}^{2}}{2 \mu_{0}}\right)_{Z} \stackrel{\leftrightarrow}{\boldsymbol{K}}\right)\right\} V_{m_{e}}$ \\
\hline $\begin{array}{l}\text { Carrying energy } \\
\qquad \Delta K+\Delta m_{m} c^{2}\end{array}$ & {$\left[\frac{h c}{2 \lambda}\right]_{X} \overrightarrow{\boldsymbol{I}} \overrightarrow{\boldsymbol{i}}$} & {$\left[\left(\frac{\boldsymbol{B}_{K}^{2}}{2 \mu_{0}}\right)_{z} \stackrel{\leftrightarrow}{\boldsymbol{K}}\right] V_{K}$} \\
\hline $\begin{array}{c}\text { Total } \\
\text { Relativistic } \\
\text { mass } \\
\text { energy } \\
\left(m c^{2}\right)\end{array}$ & & $\left.\left(\frac{\varepsilon_{0} \boldsymbol{E}^{2}}{2}\right)_{Y} \overrightarrow{\boldsymbol{J}} \overrightarrow{\boldsymbol{i}}+\left(\left(\frac{\boldsymbol{B}^{2}}{2 \mu_{0}}\right)_{Z} \stackrel{\leftrightarrow}{\boldsymbol{K}}\right)\right\} V_{m_{e}}+\left[\left(\frac{\boldsymbol{B}_{K}^{2}}{2 \mu_{0}}\right)_{Z} \stackrel{\leftrightarrow}{\boldsymbol{K}}\right] V_{K}$ \\
\hline
\end{tabular}

Given that the half-quantum $\Delta K$ momentum energy of the carrier-photon is immovably oriented perpendicularly to the $\boldsymbol{B}$ field of its own complementary electromagnetic mass increment $\Delta m_{m}$, the direction of motion of this unidirectional momentum energy is systematically determined by the orientation of its $\mathbf{B}$ field.

It is this immovable orthogonal relation that explains why unpaired electrons in ferromagnetic materials can be forced to align their spins parallel to each other in as best fit least action antiparallel mutual orientation as possible with respect to an ambient macroscopic magnetic $\boldsymbol{B}$ field, which forces their individual momentum $\Delta K$ energies to align in the same direction and add up to cause a macroscopic object such as the cylinder of the Einstein-de Haas experiment to rotate [30], or reciprocally, this is why when the unidirectional $\Delta K$ momentum energies of the carrier-photons of unpaired electrons in the ferromagnetic rod of the Barnett experiment are forced to align parallel to each other by mechanically forcing the rod to rotate, their individual $\boldsymbol{B}$ fields are also forced to align in parallel spin orientation, and add up to become measurable at our macroscopic level [30].

\section{Neutrino Emission in the Trispatial Geometry}

Interestingly, the trispatial geometry allows establishing for the first time a mechanical explanation to the emission of neutrinos. This particular solution emerges from the mandatory LC structure of elementary electromagnetic quanta in the trispatial geometry.

From this perspective, given that it is well verified that the electric charge of newly created particles mu and tau remains invariant at the same unit value as that of the electron, it can be concluded from the viewpoint provided by the trispatial geometry that the energy corresponding to the metastable excess mass that these two particles display cannot enter Y-space, because any energy increase in this space would cause by structure an increase in the value of their 
electric charge, which we know experimentally never to occur.

Given that they are massive just like the electron, they have the same LC structure as the electron in the trispatial geometry. This involves that this excess energy can exist only as a metastable increase of the energy quantum that oscillates between $\mathrm{Z}$-space and $\mathrm{X}$-space. In retrospect, the same conclusion can be hypothesized for an electron newly created by $\boldsymbol{\beta}$ - decay, which would modify the trispatial LC rest mass Equation (53) of the electron in the following manner. To simplify equations representations, we will do away from now on with the now well established unit vectors notation:

$$
m_{0+}=\left\{\left[\frac{\varepsilon_{0} \boldsymbol{E}^{2}}{2}\right]_{Y}+\left[2\left(\frac{\varepsilon_{0}\left(v_{e}+v^{\prime}\right)^{2}}{4}\right)_{X} \cos ^{2}(\omega t)+\left(\frac{\left(\boldsymbol{B}_{e}+\boldsymbol{B}^{\prime}\right)^{2}}{2 \mu_{0}}\right)_{Z} \sin ^{2}(\omega t)\right]\right\} \frac{V_{m}}{c^{2}}
$$

where $m_{0+}$ represents a slightly increased rest mass of the electron, and $\boldsymbol{v}^{\prime}$ and $\boldsymbol{B}^{\prime}$ are the slight energy increment that now momentarily oscillates between normal $\mathrm{X}$-space and magnetostatic Z-space in momentary metastable excess energy with respect to the normal stable electron rest mass energy. This solution allows the electron electric field $E$ to remain unchanged in conformity with observation.

Since this $\boldsymbol{\beta}$ - decay electron possesses slightly more energy than the well known invariant rest mass the electron, it seems quite possible that as it is in the process of leaving the destabilized neutron structure, the extreme destabilizing tensions due this initial proximity could force the electron's two neutrinic energy quanta into a violent translational motion about the X-x axis on the $\mathrm{X}-\mathrm{y} / \mathrm{X}-\mathrm{z}$ plane, that would free the two excess half-quantities momentarily in excess, causing them to escape into normal $\mathrm{X}$-space in opposite directions on this $\mathrm{X}-\mathrm{y} / \mathrm{X}-\mathrm{z}$ plane perpendicularly to the direction of motion of the electron, while the two rest energy neutrinic quantities of the oscillating half-quantum of the electron recover their usual to and fro oscillation inside the inner electron structure, that now has reached its lowest possible energy level an henceforth invariant rest mass as represented with Equation (53).

$$
m_{0+} \rightarrow m_{0}+v_{e}+\bar{v}_{e}
$$

In the trispatial geometry, the muonic and tauic neutrinos emission would obviously follow the same pattern:

$$
\begin{aligned}
& m_{0+}=\mu^{-} \\
& =\left\{\left[\frac{\varepsilon_{0} \boldsymbol{E}_{e}^{2}}{2}\right]_{Y}+\left[2\left(\frac{\varepsilon_{0}\left(v_{e}+v_{\mu}\right)^{2}}{4}\right)_{X} \cos ^{2}(\omega t)+\left(\frac{\left(\boldsymbol{B}_{e}+\boldsymbol{B}_{\mu}\right)^{2}}{2 \mu_{0}}\right)_{Z} \sin ^{2}(\omega t)\right]\right\} \frac{V_{m}}{c^{2}} \\
& m_{0+}=\tau^{-} \\
& =\left\{\left[\frac{\varepsilon_{0} \boldsymbol{E}_{e}^{2}}{2}\right]_{Y}+\left[2\left(\frac{\varepsilon_{0}\left(v_{e}+v_{\tau}\right)^{2}}{4}\right)_{X} \cos ^{2}(\omega t)+\left(\frac{\left(\boldsymbol{B}_{e}+\boldsymbol{B}_{\tau}\right)^{2}}{2 \mu_{0}}\right)_{Z} \sin ^{2}(\omega t)\right]\right\} \frac{V_{m}}{c^{2}}
\end{aligned}
$$

Resulting in similar mu and tau neutrinos emissions: 


$$
m_{0+}=\mu^{-} \rightarrow m_{0}+v_{\mu}+\bar{v}_{\mu} \text { and } m_{0+}=\tau^{-} \rightarrow m_{0}+v_{\tau}+\bar{v}_{\tau}
$$

Of course, $\boldsymbol{\beta}+$ decay, anti-muon and anti-tau will result in identical neutrino emissions, leaving behind a single positron instead of an electron.

The fact that both neutrinos produced during each emission can only be released as an identical pair moving in opposite directions perpendicularly to the direction of motion of the emitting particle, makes it impossible for neutrinos produced by decaying muons coming in direct line from the Sun's surface in the general direction of the detector to be detected since they escape and move on planes perpendicular to the Sun-detector axis.

So according to the trispatial characteristics of neutrino emission, the only neutrinos/antineutrinos that can possibly be detected originating from the Sun will be a small fraction of those released by decaying muons in motion on a plane perpendicular to the Sun-detector axis, that is, mainly neutrinos emitted at the outside limits of the visible disk of the Sun, which is a conclusion that would go a long way in explaining why their detection rate has consistently remained so far below what current theories predict.

This conclusion could easily be verified experimentally by focusing detection equipment directly to the circumference of the solar disk.

Finally, since they escape as simple momentum related unidirectional kinetic energy amounts in X-space, deprived of the complementary transverse electromagnetic component oscillating between $\mathrm{Y}$-space and Z-space that explains omnidirectional inertia as perceived from normal X-space, that is "electromagnetic mass", as well as "electric charge", for all electromagnetic elementary particles in the trispatial geometry, this provides a clear explanation of why no mass nor charges were ever detected for them in all experiments where they were involved.

\section{Up and Down Quarks in the Trispatial Geometry}

The last particles that must be examined before resonance states can be addressed are the up and the down quarks that have been confirmed to be the only scatterable point-like behaving electromagnetic charged massive elementary subcomponents that can be identified in protons and neutrons, during extensive non-destructive scattering experiments carried out from 1966 to 1968 at the SLAC facility [12] [18] [31].

The trispatial mechanics of creation of protons and neutrons from the only two possible combinations of triads of electrons and positrons interacting in close enough proximity without sufficient momentum energy to escape each others' mutual capture is described in reference [18].

Given that up and down quarks always display the same point-like behavior as electrons and positrons during all such scattering experiments with electrons or positrons, it has long been suspected in the community that these up and down quarks making up the inner scatterable structure of nucleons could possibly be 
positrons and electrons whose masses and charge characteristics would be warped into these potentially altered states by the stresses imposed by these most energetic least action equilibrium states that these particles can possibly reach in Nature [10] [12] [18].

This possibility immediately brings to light a possible explanation to the observed fact that no up or down quark could ever be observed moving separately in space after having been scattered out of a nucleon by sufficiently energetic scattering. Indeed, it they are electrons and positrons whose characteristics are warped into those observed for up and down quarks by their intensely stressed nucleonic electromagnetic environments, they would of course immediately recover their normal electron or positron characteristics as soon as they escape these warping stresses.

The specific electron and positron characteristics that would be warped out of skew by these intense stresses are first of all their masses, which have been determined to lie between 1 and $5 \mathrm{MeV} / \mathrm{c}^{2}$ for the up quark and between 3 and 9 $\mathrm{MeV} / \mathrm{c}^{2}$ for the down quark, and their electric charges that have been determined to be $2 / 3$ of the charge of the positron for the up quark, and $1 / 3$ the charge of the electron for the down quark ([32], p. 382).

It so happens that the trispatial geometry allows defining a clear mechanics of creation of nucleons from the only two possible combinations of triads of electrons and positrons, which provides a logical explanation to these stress induced changes in characteristics, and also on the nature of these electromagnetic stresses [18].

In the trispatial geometry, the mass and charge of stable elementary particles vary as an inverse function of each other as a function of their distance from the coplanar Y-Z axis within electrostatic Y-space [12] [18].

The distance from the $\mathrm{Y}-\mathrm{z}$ axis within $\mathrm{Y}$-space at which an electron-positron pair decouples from a destabilized $1.022 \mathrm{MeV}$ photon is by structure $3.861592641 \mathrm{E}-13 \mathrm{~m}$ [17], which corresponds to the electron "classical radius" divided by the fine structure constant $\left(r^{\prime}=r_{e} / \alpha\right)$.

At this distance from the $\mathrm{Y}-\mathrm{z}$ axis, its charge exactly corresponds to the well known unit charge of $1.602176462 \mathrm{E} 019 \mathrm{C}$ and mass of $9.10938188 \mathrm{E}-31 \mathrm{~kg}$. These values well established experimentally allow determining the corresponding values for the up and down quarks as in Table 2 [18], which fall precisely within the experimentally estimated limits for these masses.

This allows establishing the following general equation to calculate the invariant effective masses of the only three stable massive and electrically charged elementary electromagnetic particles, which are behaving point-like in all scattering encounters, that are the only electromagnetic elementary sub-components of all atoms that exist in the universe, by means of the electrostatic energy induction constant $K=1.220852596 \mathrm{E}-38 \mathrm{j} \cdot \mathrm{m}^{2}$ established from the Coulomb equation in references [12] [17] [18]. Of course, the positron can be considered as being identical to the electron except for the sign of its charge. 
Table 2. Relation between up and down quarks charges and masses with respect to their distance from the $\mathrm{Y}-\mathrm{z}$ axis within electrostatic $\mathrm{Y}$-space.

Table of the effective charges and masses of the electron, the up quark and the down quark, estimated on the assumption that the unit charge of the electron would be the amount of charge induced at the distance from the $\mathrm{Y}-\mathrm{z}$ axis at which electron-positron pairs separate during the pair production process.

\begin{tabular}{cccc}
\hline Particle & $r^{\prime}=r_{e} / \alpha$ & Charge & mass \\
Electron & $r_{e}^{\prime}=3.861592641 \mathrm{E}-13 \mathrm{~m}$ & $1.602176462 \mathrm{E}-19 \mathrm{C}$ & $9.10938188 \mathrm{E}-31 \mathrm{~kg}$ \\
Up quark & $r_{e u}^{\prime}=2.574395094 \mathrm{E}-13 \mathrm{~m}$ & $1.068117641 \mathrm{E}-19 \mathrm{C}$ & $2.04961092 \mathrm{E}-30 \mathrm{~kg}$ \\
Down quark & $r_{e d}^{\prime}=1.287197547 \mathrm{E}-13 \mathrm{~m}$ & $5.340588207 \mathrm{E}-20 \mathrm{C}$ & $8.19844378 \mathrm{E}-30 \mathrm{~kg}$ \\
\hline
\end{tabular}

$$
m_{i[d, u, e]}=K\left(\frac{3 \alpha}{n r_{0} c}\right)^{2} \quad(n=1,2,3)
$$

In the trispatial geometry, this lessening of the charges of the up and down quarks due to the electromagnetic stresses they are subjected to inside nucleons cannot occur however without being compensated by an increase of the magnetic field of the particle and of its carrier-photon, as is demonstrated for the magnetic drift suffered by the electron carrier-photon energy, even as far from the nucleus at the ground state of the hydrogen atom [23].

Given that the up and down quarks stabilize at such precise relative distances from the $\mathrm{Y}-\mathrm{z}$ axis in the trispatial geometry, it becomes possible to establish their magnetic drift constants from these distances:

$$
S_{U}=\frac{r_{e u}^{\prime}}{r_{e}^{\prime}}=\frac{2}{3} \text { and } S_{D}=\frac{r_{e d}^{\prime}}{r_{e}^{\prime}}=\frac{1}{3}
$$

These magnetic drift constants and wavelengths now allow establishing the trispatial LC equations of both up and down quarks:

$$
\begin{aligned}
& m_{U}=\frac{E_{U}}{c^{2}}=\frac{1}{c^{2}}\left\{S_{U}\left[\frac{h c}{2 \lambda_{U}}\right]_{Y}+\left(2-S_{U}\right)\left[2\left(\frac{\left(e^{\prime}\right)^{2}}{4 C_{U}}\right)_{X} \cos ^{2}(\omega t)+\left(\frac{L_{U} i_{U}^{2}}{2}\right)_{Z} \sin ^{2}(\omega t)\right]\right\} \\
& m_{D}=\frac{E_{D}}{c^{2}}=\frac{1}{c^{2}}\left\{S_{D}\left[\frac{h c}{2 \lambda_{D}}\right]_{Y}+\left(2-S_{D}\right)\left[2\left(\frac{\left(e^{\prime}\right)^{2}}{4 C_{D}}\right)_{X} \cos ^{2}(\omega t)+\left(\frac{L_{D} i_{D}^{2}}{2}\right)_{Z} \sin ^{2}(\omega t)\right]\right\}
\end{aligned}
$$

The carrier-photon of each up and down quark within nucleons would of course have the same internal trispatial LC structure as that of the electron carrier-photon, that is, that shown with Equation (55), and would relate to its carried particle in the same manner, as described in Table 1 for the electron in motion, the only differences being the immensely higher energy levels that these nucleonic carrier-photons reach, and the amount of magnetic drift that they themselves suffer from the stress imposed by the nucleonic electromagnetic environment [18]. 
This relation between each up quark and each down quark with each its own carrier-photon makes them amenable to being represented by the wave function in a manner similar to that of the electron in the hydrogen ground state, as we will see further on.

\section{Parallel and Anti-Parallel Relative Magnetic Spin Orientations}

In Quantum Mechanics (QM), the concept of "spin" is so weakly related to the magnetic field that although it is technically associated to the magnetic moment of charged particles, even this magnetic moment is seen by most in the community as a simple mechanical angular momentum $(S z= \pm 1 / 2 \hbar)$ with no clear reminder that it very specifically concerns the relative magnetic polarities orientation, either parallel or anti-parallel, of the magnetic fields of elementary electromagnetic quanta relative to each other. For all practical purposes, it is perceived as a mechanical "spinning motion" in two possible transverse directions perpendicularly to the direction of motion in the classical mechanics sense with no real relation with electromagnetism.

However, the very idea of a "magnetic spin" of elementary particles being akin to the classical/relativistic mechanics concept of "angular momentum" directly clashes with the experimentally confirmed fact previously mentioned that no unbreachable limit was ever detected at any distance from electrons' centers, however close two electrons came to each others' centers during absolutely all scattering encounters, because the very idea of an "angular momentum" implies the existence of a volume that can rotate, which is meaningless in the case of an elementary electromagnetic quantum such as the electron, for which no volume can be measured since it systematically behaves point-like in all scattering encounters.

The disconnect between the QM concept of "spin” and the physical relative polar magnetic orientations of elementary electromagnetic quanta is so important that many remain convinced that "spin" would be an "intrinsic" angular momentum property of particles, instead of what it can only be, that is a "relative" property that remains meaningless unless at least two electromagnetic quanta are involved, which is the uncircumventable condition for the very ideas of "magnetic parallel orientation" and "magnetic antiparallel orientation" to make any sense.

The fact that two electrons succeed so easily in associating in a very strong and intimate least action antiparallel magnetic spin covalent bounding to unite two hydrogen atoms into an $\mathrm{H}_{2}$ molecule despite their electric repulsion function of the inverse square of the distance, reveals that an interaction law of a higher order than the inverse square Coulomb force is simultaneously at play to so easily initiate and maintain such a powerful least action magnetic covalent bound between two electrons.

Interestingly, experiments carried out as recently as 2014 by Kotler et al. [33] experimentally demonstrated that the interaction law involved, when 2 electrons are forced to interact in parallel magnetic spin alignment, is the inverse cube in- 
teraction law function of the distance, which is the interaction that overcomes the inverse square repulsion Coulomb law when two electrons are forced to come sufficiently close to each other in antiparallel magnetic spin alignment. The relation between these two interaction laws is described in Figure 6.

Also, an experiment carried out in 1998 already confirmed this magnetic interaction function of the inverse cube between magnets having the same magnetic field configuration as that of elementary electromagnetic quanta, which allowed analyzing this magnetic interaction law in relation with the oscillating nature of the magnetic energy of electromagnetic quanta revealed in the trispatial geometry [34], that brought to light the fact that the magnetic fields of elementary electromagnetic quanta behave at any given moment like magnetic monopoles that constantly reverse their polarity as a function of time according to their energy frequency [11].

This conclusion finally brings to attention the key function of the relative frequency ratios existing between elementary electromagnetic quanta in explaining why two electrons can so easily magnetically stabilize in covalent bounding despite their repelling same electric charges signs, due the ratio 1 of the synchronous frequencies of the spherical expansion and regression of their respective magnetic energies presence; also why an electron and a positron captive in metastable positronium configuration can combine to convert to electromagnetic photon states precisely due to the ratio 1 of their synchronous magnetic reversal frequencies combined with their attracting opposite electric charges signs [11]; and finally why an electron and a proton can so systematically magnetically repel each other to end up stabilizing at the known electron ground state orbital mean distance despite their attracting opposite electric charges signs [11] [34], due to the asynchronous frequencies ratio of the spherical expansion and regression of their magnetic energy presence, whose mechanics was summarily analyzed in

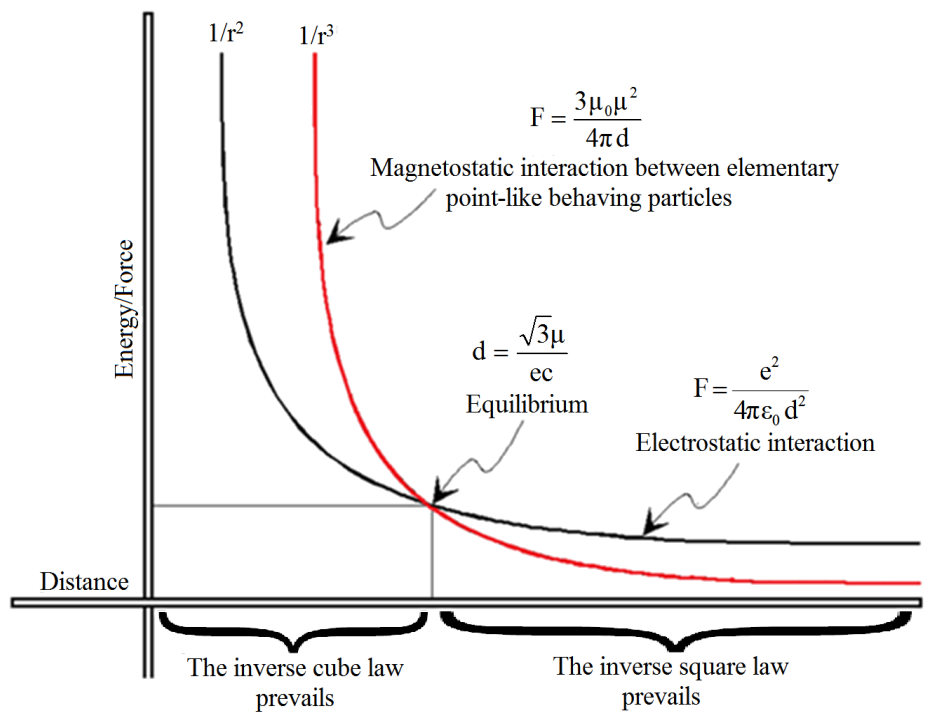

Figure 6. Intersecting inverse square and inverse cube interaction curves. 
references [10] [11] [34], and that will be analyzed in more details further on in relation with the resulting resonance states.

But let us first analyze how the asynchronous magnetic interaction between the invariant frequency of the energy of the electron rest mass and the variable frequency of the energy of its carrier-photon allow both quanta to define the irregular resonance state known under the name of Zitterbewegung of the electron in motion.

\section{Zitterbewegung}

Considering Table 1 again that puts in perspective the fact that the electron in motion involves two different energy quanta, that not only electromagnetically oscillate at different frequencies, but whose harmonic oscillation centers $\otimes$ are physically separated by structure on a plane transverse with respect to the direction of motion of the system in space (see Figure 7).

Comparing the electron rest mass Equation (53) with its carrier-photon Equation (55) indeed shows that each quantum possesses its own trispatial junction $\otimes$ which are separated by structure from the simple fact that their energy oscillates between different pairs of spaces in the trispatial complex, that of the electron oscillating between Z-space and X-space, while that of its carrier-photon oscillates between Z-space and Y-space, on top of oscillating at different frequencies. This means that except for the case when the carrier-photon would possess exactly $0.511 \mathrm{MeV}$ of energy, both components of the electron in motion are physically unable to associate in exactly synchronized attractive relative anti-parallel magnetic spin alignment, which highlights the contrast between these predictable and measurable asynchronous resonance interactions, and the unpredictable spontaneous stochastic fluctuations of the zero point energy level of QFT currently assumed to be responsible for Zitterbewegung.

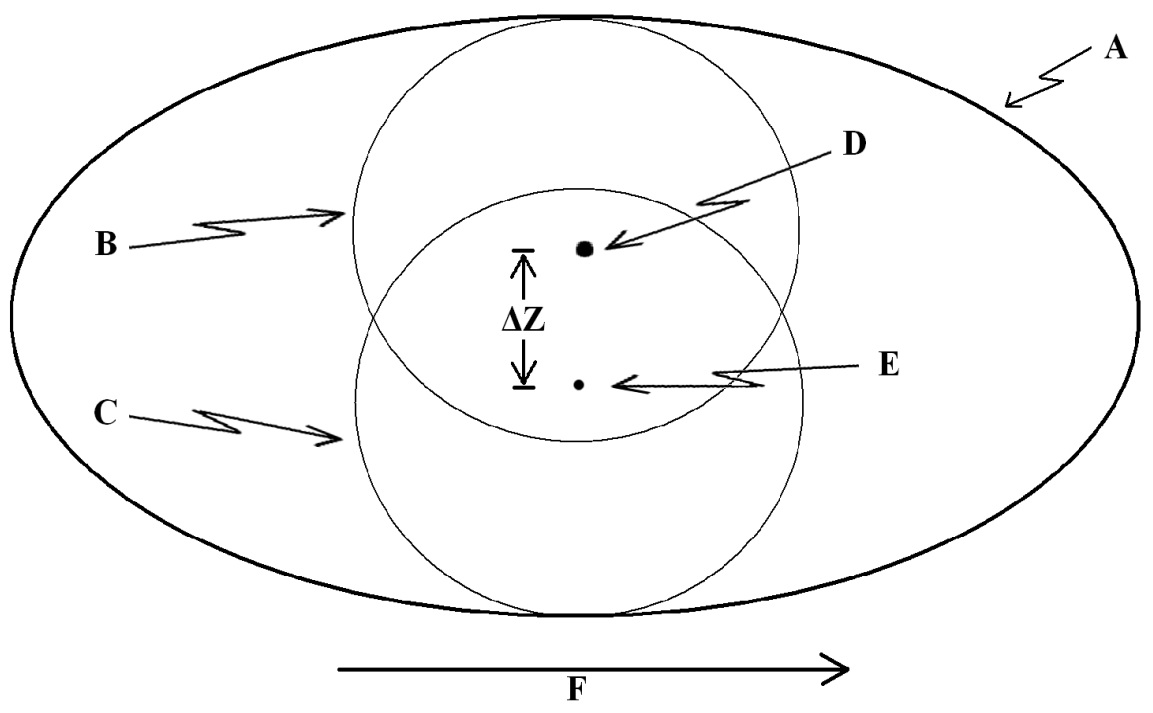

Figure 7. Free moving electron. 
In reality, any difference in frequency between both components can only force both trispatial junctions to follow oscillating trajectories that seems in appearance erratic transversally with respect to the direction of motion of the twin component system, due to the uninterrupted asynchronous sequence of cyclic alternance between attractive anti-parallel spin alignment states and repulsive parallel spin alignment states, which can only generate the resonance state that came to be identified as the Zitterbewegung of the moving electron.

We will see further on that a third oscillation process, axial within atomic structures this time, will become involved when the electron is captured in least action electromagnetic equilibrium in atomic orbitals, which generates the actual three-components complex resonance volume within which de Broglie concluded the electron has to be captive into in the hydrogen atom and that Schrödinger meant to describe with the wave function.

Legends of Figure 7:

A. Symbolic representation of the resonance volume of the electron energy in free motion as definable by a wave function involving the cyclic magnetic spins reversal interaction within Z-space of both electromagnetic quanta of the electron in motion "B" and "C", to be correlated with the resonance mechanics symbolically represented in Figure 8 and Table 1.

B. Symbolic representation of the spherical volume of the oscillating magnetic energy of the electron in Z-space. Ref. Figure 5(c) as applied to the electron inner oscillating structure and Equation (53). This volume corresponds to its magnetic energy varying from zero presence to maximum presence calculated with Equation (22), and to half its invariant mass as determined in reference [14].

C. Symbolic representation of the spherical volume of the oscillating magnetic energy of the electron carrier-photon in Z-space. Ref. Figure 5(c) as applied to the carrier-photon inner oscillating structure and Equation (55). This volume corresponds to its magnetic energy varying from zero presence to a maximum presence calculated with Equation (23), and to the velocity related electron magnetic mass increment $\Delta \mathrm{m}_{\mathrm{m}}$ as calculated with Equation (10). This volume also corresponds to the energy contained in the volume defined by the Schrödinger wave function.

D. Central resonance anchoring point $\otimes$ of the magnetic energy of the electron within resonance volume " $\mathrm{A}$ ", that is, its trispatial junction point, where the origin of the trispatial complex is located for the electron energy quantum (Figure 4).

E. Central resonance anchoring point $\otimes$ of the magnetic energy of the electron carrier-photon within resonance volume " $A$ ", that is, its trispatial junction point, where the origin of the trispatial complex is located for the carrier-photon energy quantum (Figure 4).

Note that more realistically, the combined magnetic volume of both magnetic quanta should amount to a single spheroid whose dimensions would vary as a function of the constantly varying sum of the magnetic energies present in 


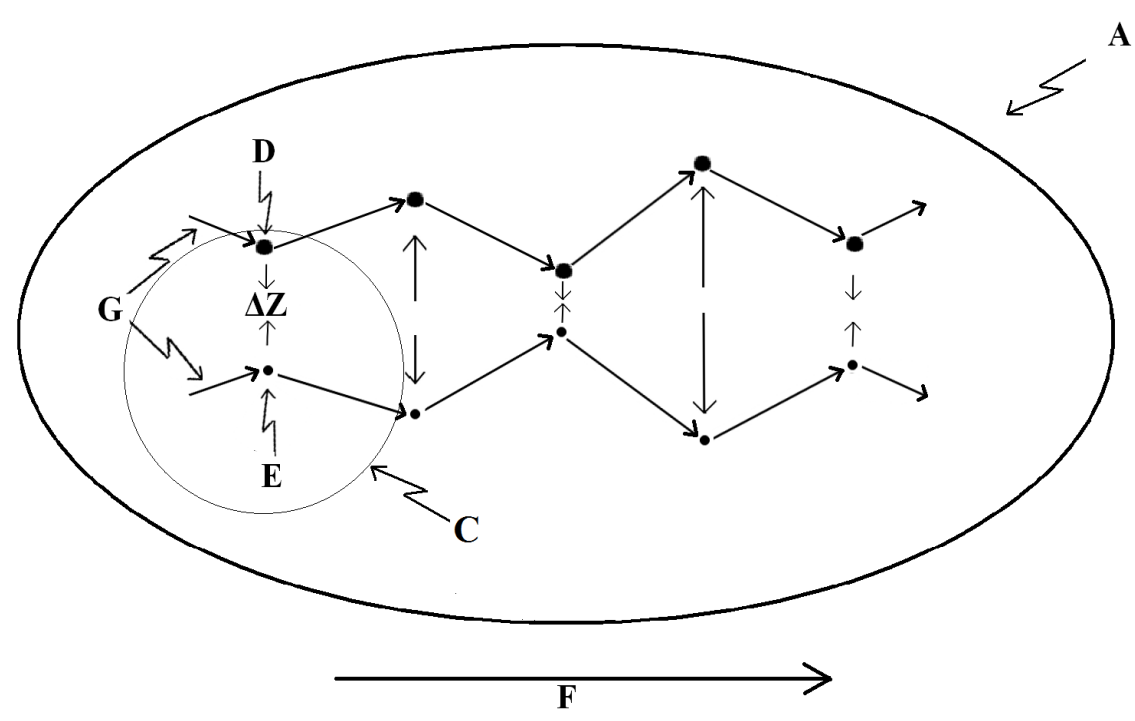

Figure 8. Zitterbewegung.

Z-space at any given instant due to their constant alternance between maximum presence and zero presence at different frequencies, and within which both anchoring points "D" and "E" would physically remain at some varying distance $\Delta Z$ from each other by structure as they oscillate toward and away from each other as will be analyzed with Figure 8. This exploded representation is only meant to help visualize that both quanta oscillate separately at their respective frequencies.

F. Unidirectional orientation in $\mathrm{X}$-space of the $\Delta \mathrm{K}$ electron momentum motion energy.

$\Delta Z$-Zitterbewegung distance between both trispatial junctions " $D$ " and "E".

Figure 7 should be correlated with Figure 8 that represents the transverse interplay determining the actual Zitterbewegung resonance state.

Additional legend for Figure 8, completing those defined for Figure 7:

G. The transverse Zitterbewegung resonance oscillation resulting from the cyclic relative spin reversal of the magnetic energy spheres " $\mathrm{B}$ " and " $\mathrm{C}$ " according to their respective frequencies (see Figure 7) result in an uninterrupted sequence of successive closing in toward each other and moving away from each other of both central resonators anchoring points " $D$ " and "E". The irregularity of the successive cyclic reversed distances is only meant to highlight that both magnetic spheres of Figure 7 cycle from maximum presence to zero presence at different frequencies, resulting in an irregularity of resonance cycles that generate the observed Zitterbewegung.

As a matter of fact, the relative motion freedom of both tri-spatial junctions with respect to each other can only be perpendicular to the direction of motion of the system, since the stability by structure of the amount of translational energy of the carrier-photon at any given moment depends uniquely on the Coulomb interaction between the carried electron and other charged particles. This constraint thus prevents any longitudinal deceleration or acceleration relative to each other from being involved in their motion. 
The only remaining possible direction of motion available for the two trispatial junctions with respect to each other is thus transverse with respect to the direction of motion of the system, which implies that at any given moment, both trispatial junctions will be at distance $\Delta Z$ (Zitterbewegung distance) from each other (see Figure 7), computable as a function of the state of the electromagnetic harmonic oscillations parameters of both quanta at this moment.

Haven't we just identified here the cause of the "Zitterbewegung" that Schrödinger was discussing in his analysis of the Dirac wave equation [7] and that he found amounted to an irregular circular fluctuating motion of the electron, which is superimposed to its translational motion? The difference with Schrödinger's description is that while QM asserts that the magnetic spin moment is caused by the Zitterbewegung motion (observed, but unexplained), the trispatial geometry approach predicts and mechanically explains that it would be the Zitterbewegung which would be due to the forced interaction between the pre-existing cycling magnetic energy of the electron and the pre-existing cycling magnetic energy of its carrier-photon, due to their frequencies differences.

So, on top of revealing that the actual resonance volume "visited" by the two interacting oscillating electromagnetic quanta of the electron in motion will vary with the varying frequency of the increasing or decreasing energy of the carrier-photon due to the varying proximity of the carried electron with other charged particles in its environment, this analysis reveals that when the energy of the carrier-photon becomes exactly equal to that of the invariant rest mass of the electron, that is $0.511 \mathrm{MeV}$, the amplitude $\Delta \mathrm{Z}$ of the Zitterbewegung oscillation will fall to zero due to perfectly coinciding anti-parallel magnetic spin alignment while the resonance volume synchronizes in simple harmonic oscillation, which could be verified experimentally.

\section{The Wave Function and the Resonance State of the Moving Electron}

This brings us to put in perspective the resonance volume defined as the moving electron's fixed amount of harmonically oscillating energy interacts with the varying amount of its harmonically oscillating carrier-photon energy, as the electron moves in space, with respect to the traditional form of the wave function being used to represent it.

As mentioned at the beginning of this paper, the wave function was initially introduced to represent the resonance volume that de Broglie had concluded that the electron had to be captive into when stabilized in the hydrogen ground state [1]. The method was then extended to represent electrons and electromagnetic photons in free motion.

As it currently stands, the Schrödinger wave function involves the complex harmonic oscillation of an unclearly defined single resonator mathematically combining a real and an imaginary part, whereas from the trispatial geometry perspective, we observe that the electron in motion involves two very clearly defined electromagnetic resonators in separate simple harmonic oscillation. 
Even if as it stands, although the Schrödinger wave function allows accounting for the complete complement of momentum energy $\Delta \mathrm{K}$ of the electron in motion or captive in atomic orbitals, it is unable to account for the electron Zitterbewegung as stemming from the electromagnetic properties of the electron and of its carrying energy.

Indeed, its classical mechanics origin incompletely related to electromagnetism does not allow reverse engineering any of the electromagnetic characteristics of the resonating electron from this wave function characteristics, which is the disconnect that Feynman observed in 1964 that prevented Quantum Mechanics from being completely synchronized with electromagnetism [35]:

"There are difficulties associated with the ideas of Maxwell s theory which are not solved by and not directly associated with quantum mechanics... when electromagnetism is joined to quantum mechanics, the difficulties remain."

Let us note here that reverse-engineering the manner in which observed phenomena can be explained is a quite usual method of exploration in scientific circles. Indeed, it possibly is the only effective method, but its minimal condition of success hinges on considering as few arbitrary axiomatic grounding premises as possible, while taking into consideration as many related confirmed experimental observations as can be gathered, and finally no unrelated element.

Faced with this dead end when starting from the wave function characteristics, it appeared logical to attempt reverse-engineering the electromagnetic resonating structure of the electron and of its carrier-photon, not from the characteristics of the wave function as de Broglie attempted to do, but from the well established and well known characteristics of electromagnetic energy, which led to the present solution elaborated from the trispatial geometry perspective.

To get an idea of the challenge that de Broglie was confronted with, let's examine how the nature of the resonator generating a resonance volume well understood in classical mechanics can rather easily be understood by means of reverse-engineering.

Who has not observed with a modicum of curiosity how a guitar string that has just been picked practically "disappears" from sight, particularly in the middle of its length as it vibrates, while transversally "visiting", so to speak, a very characteristic volume of space, which is its actual "resonance volume" that can be represented by a wave function?

In this case, we obviously know in advance that the resonator is a continuous elastic string tied at both ends, because we can actually see the string when at rest, and even though it seems to disappear when vibrating, we also know that the string still physically exists even if we don't see it as it momentarily oscillates transversally too fast for us to see.

We can also imagine that someone having never seen a guitar nor any other string instrument, but expert in mathematics, being shown the very characteristic wave function describing completely the stationary resonance volume of the 
string, after carefully observing the symmetrically diminishing toward zero of the amplitude of the resonance volume on either side of its maximum value, may well be able to deduce that this resonance volume could only have been produced by a continuous elastic string anchored in fixed positions at both end, thus discovering and understanding the nature of a resonator that he knew nothing about previously.

But no such luck with the Schrödinger's wave function because, as we saw in the previous section, the electromagnetic resonance anchoring points of his wave function that allow understanding how its resonance mechanics can be established, are not conveniently located outside the resonance volume as in the case of the guitar string, but inside this volume, which provides no clue whatsoever that could help even recognizing their very existence and consequently their relation to electromagnetism. This is why the only possible reverse engineering direction that could reveal the relations between Schrödinger's wave function and electromagnetism was from the confirmed characteristics of electromagnetic energy.

In fact, the identification of the electromagnetic localizations parameters allowed by the trispatial mechanics shows that the Schrödinger wave function has been mapping the resonance volume of the $\Delta K$ momentum related half-quantum of the electron carrier-photon, which means that when the wave function is made to theoretically collapse, it is the momentary location in space of the electron carrier-photon trispatial junction "E" which is physically located [36], and its momentary $\Delta K$ momentum energy which is revealed (see Figures 7-9).

The relative position of the electron trispatial junction " $D$ " can then be established to lie at distance $\Delta Z$ (momentary Zitterbewegung distance between both trispatial junctions) from the carrier-photon trispatial junction " $E$ " at the same perpendicular distance from the atomic nucleus when the electron is captive in an atomic orbital (see Figure 9).

With the help of Figure 7 to establish a mental representation of the related "B" /"C" magnetic interactions, we thus observe that both electromagnetic components are kept together by the sequence of cyclic transverse magnetic attraction/repulsion reversals due to the fact that their separate magnetic energy " $\mathrm{B}$ " and "C" are constantly switching between mutual relative parallel and anti-parallel alignments of their magnetic spins at different frequencies [10] [11] [14] [27]; the spherical magnetic orientation of the electron energy " $B$ " cyclically reversing at the invariant frequency calculated with Equation (15), while that of its carrier-photons " $\mathrm{C}$ " that varies with the amount of kinetic energy of which it is made, cyclically reverses at the frequency that can be calculated with Equation (14).

Each closing in sequence between the " $D$ " and "E" trispatial junctions corresponds to the duration of a phase of magnetic antiparallel alignment of the spins of both magnetic spheres " $\mathrm{B}$ " and " $\mathrm{C}$ ", corresponding to the fact that the sum of their energies present in Z-space progressively diminishes toward some momentary minimum presence value, while each moving away sequence corresponds 


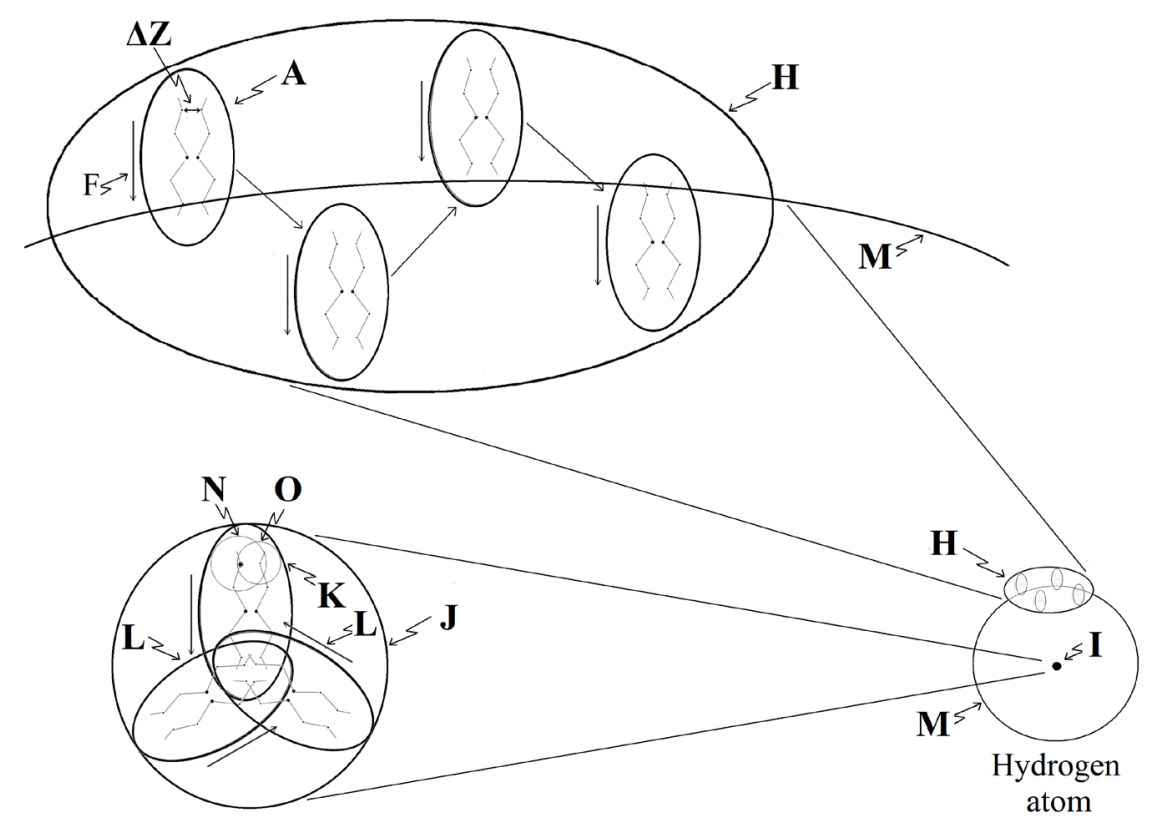

Figure 9. The hydrogen atom resonance states.

to a phase of magnetic parallel alignment of their spins, corresponding to the fact that the sum of their energies present in Z-space progressively increasing toward some momentary maximum presence value.

Given that both magnetic spheres oscillate at different frequencies, these minima and maxima will vary according to the extended resonance sequence specific to their combination as a function of the variation of the adiabatic energy making up the carrier-photon as it moves in space with reference to varying distances between this electron in motion and surrounding other charged particles, thus completely accounting for the apparently random observed Zitterbewegung.

\section{The Resonance States of the Electron in Atomic Orbitals}

As analyzed in references [10] [11], the only way for an electron to be stopped in its motion when moving freely in Nature, is for it to be captured into some least action axial electromagnetic equilibrium states in one of the authorized orbitals in an atom.

During its just analyzed free motion already, both separate electromagnetic quanta making up the electron in motion, that is, that of the invariant energy of its rest mass " $D$ " and that of the energy of its carrier-photon " $E$ ", can only be maintained together because the interaction in high frequency cyclic inversion of their magnetic energy " $B$ " and " $C$ ", whose attractive presence phases, despite being intermittent and asynchronous as a function of the inverse cube of the distance, is sufficiently strong at so short distances, to insure a cohesion that can only be a state of least action by definition.

But as powerful this interaction can be at so short distances between the mag- 
netic energy spheres " $B$ " and " $C$ ", it is dwarfed out of all proportions with respect to the power of the interaction between these magnetic energy spheres and the magnetic energy spheres " $N$ " of the carrier-photons of the up and down quarks making up the proton constituting the nucleus of a hydrogen atom (see Figure 9 and Figure 10).

So powerful in fact, that even at the "relatively astronomical" approximate distance of $5.29 \mathrm{E}-11 \mathrm{~m}$ from the proton, the complex resultant of their combined cyclic repulsive parallel magnetic interaction is sufficient to literally stop the electron in its tracks as it is in the final leg of its acceleration motion toward the proton, the latter due to the Coulomb force attraction between its negative charge and the positive combined charges of the three quarks, and that the complex resultant of their combined cyclic attractive antiparallel magnetic interaction is sufficient to keep it from escaping and keep it captive in a stabilized least action axial electromagnetic equilibrium state.

The parameters of Table 2 and Table 3, and Equations (61) to (64) indeed allowed calculating in references [10] [11] [18] [34] that the magnetic energy component " $\mathrm{N}$ " of the energy of each of the quarks' carrier-photons is more than 600 times more powerful than that of the invariant magnetic energy of the rest mass of the electron " $\mathrm{B}$ ", which mutually build up their combined strength to about 2000 times that of the electron and its carrier photon.

During the actual stopping process, the forward moving $\Delta K$ momentum related energy half-quantum " $F$ " of the electron carrier-photon had no option, due to its forward inertia, but to escape as a well known bremsstrahlung electromagnetic photon, whose energy amount is $13.6 \mathrm{eV}$ in the case of the establishment of the electron in the hydrogen ground state orbital " $\mathrm{H}$ ".

As this momentum energy escapes, the exact same amount of replacement $\Delta K$ momentum energy " $\mathrm{F}$ " is simultaneously adiabatically re-induced by the Coulomb force as described in reference [10], because it is well verified that Coulomb interaction between charges forbids that an amount different from $27.2 \mathrm{eV}$ be induced as a carrier-photon in unit charges separated by this distance of $5.29 \mathrm{E}-11 \mathrm{~m}$.

This new half-quantum of $\Delta K$ momentum energy " $F$ " now directly and unswervingly oriented by structure toward the proton will continue applying a continuous "pressure" to keep the electron negative charge moving toward the oppositely signed resultant of the charges of the nuclear subcomponents, even if its forward motion is impeded by the magnetic counter pressure existing between its magnetic energy " $\mathrm{B}$ " and that of the inner proton carrier-photons " $\mathrm{N}$ ".

And it is the pressure/counter-pressure interplay between the electron carrier-photon momentum energy $\Delta K$ "F" and the complex interaction between the oscillating magnetic spheres " $\mathrm{B}$ " and " $\mathrm{N}$ " involved that determine the resonance volume described by the Schrödinger wave function, as we will see.

It must be said that the capture of an electron by a proton to form a hydrogen atom is possibly the best-understood process involving elementary particles stabilized into least action axial electromagnetic equilibrium. It has however been 


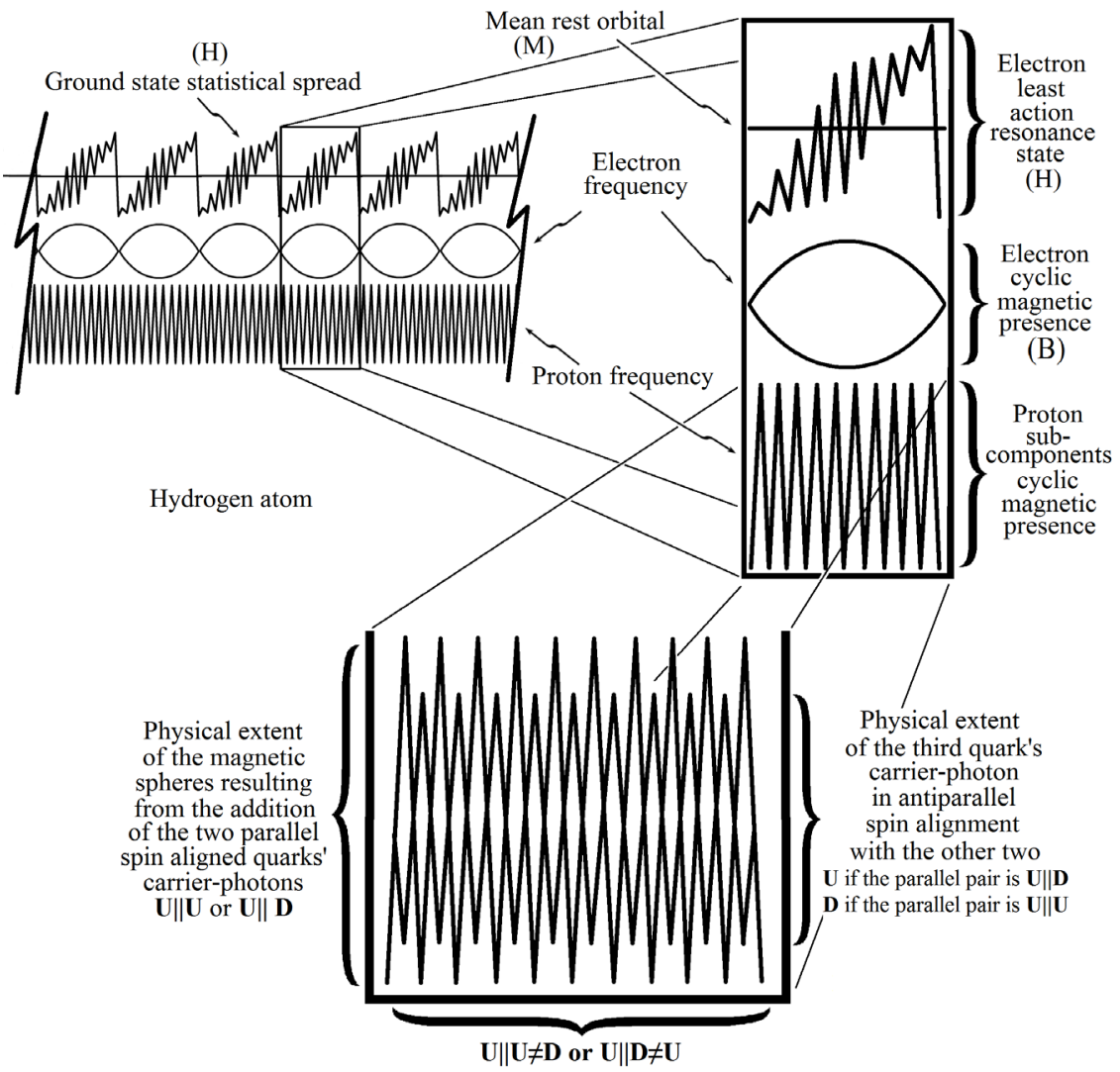

Figure 10. Establishment of the least action resonance state of the electron in the hydrogen atom.

Table 3. Energies and wavelengths of the rest masses of the up and down quarks.

\begin{tabular}{|c|c|c|c|}
\hline \\
\hline \multicolumn{4}{|c|}{$\begin{array}{l}\text { Table of the energies and wavelengths of the effective masses of the up and down quarks, estimated } \\
\text { on the assumption that the unit charge of the electron would be the amount of charge induced at } \\
\text { the distance from the Y-z axis at which electron-positron pairs separate during the pair production } \\
\text { process. } \\
\text { Particle }\end{array}$} \\
\hline \multicolumn{4}{|c|}{$r_{e}^{\prime}=3.861592641 \mathrm{E}-13 \mathrm{~m}$} \\
\hline & & $1.149747531 \mathrm{MeV}$ & $1.078360096 \mathrm{E}-12 \mathrm{~m}$ \\
\hline Down quark & $r_{e d}^{\prime}=1.287197547 \mathrm{E}-13 \mathrm{~m}$ & $4.598990173 \mathrm{MeV}$ & $2.69590021 \mathrm{E}-13 \mathrm{~m}$ \\
\hline
\end{tabular}

studied and understood for the past century only by means of the two traditional distinctly different filtering paradigms that cannot be directly reconciled, that of classical/relativistic mechanics physics and that of quantum mechanics physics.

From the classical/relativistic mechanics paradigm, inherited from Newton's mechanics, stabilization of the electron at the calculated distance of $5.291772083 \mathrm{E}-11 \mathrm{~m}$ can be related only to the idea that the electron would be a localized mass without internal structure orbiting the proton at this distance at the velocity corresponding to its $\Delta K$ momentum energy, a velocity that can be calculated either from the classical or the relativistic viewpoint depending on whether or not the gamma factor is taken account of in its calculation. 
From this perspective, it is not conceivable that the electron could conserve its $\Delta K$ momentum kinetic energy as calculated with Equation (11) if it were to slow down and become immobile at this axial distance from the proton, because the very existence of kinetic energy, from the classical/relativistic mechanics perspective, depends on the velocity of a massive body [11]. From this perspective, if a massive body were to slow down in this manner, its kinetic energy is deemed to convert to an equivalent amount of "potential" energy, which would be tantamount to depriving the electron of any possibility of remaining "in orbit", and it is considered that this would lead to the electron theoretically "falling" onto the proton.

But of course, since we know with certainty that this never happens in physical reality, from countless experiments carried out over the course of the past century, we also know that this conclusion, drawn with reference to macroscopic massive bodies before the existence of electric charges and of the Coulomb force were discovered, is somehow at least partly misleading when applied to the behavior of electric charges, even if it appears satisfactory when applied to massive bodies at our macroscopic level.

From the quantum mechanics perspective, inherited from the establishment of the Schrödinger wave function and Heisenberg's statistical distribution in the 1920 's, the electron stabilized in the hydrogen ground state is seen with $100 \%$ probability as being present within a clearly defined resonance volume of space about the proton, within which the energy of the electron, without any internal structure just like in classical/relativistic mechanics, is estimated to statistically be more concentrated (or more often present) about this mean distance of $5.29 \mathrm{E}-11 \mathrm{~m}$ from the proton, a volume within which the electron cannot be seen as moving on a clear trajectory contrary to classical/relativistic mechanics, even though it is clearly established that it can be axially located anywhere within this volume when any theoretical wave function collapse is calculated, and that its most probable location tends to coincide with the classical Bohr orbit representation, which is expressed as a probability of increased density of the energy of the electron within the volume described by Heisenberg's statistical method.

Its total energy is defined in more general terms with the Hamiltonian inherited from the classical mechanics paradigm as combining in a single conservative concept the sum of the kinetic energy and the potential energy that accounts for is momentum in classical/relativistic mechanics, intriguingly still internally grounded on the same $p=m v$ Newtonian conservative momentum concept $(\mathrm{p}=$ $\gamma \mathrm{mv}$ from the relativistic perspective), that causes the $\Delta K$ amount of related kinetic energy to still depend on velocity, even if no velocity can be associated with the spread out energy of the electron as currently represented by the wave function resonance volume.

Although both traditional paradigms take into account the $\Delta K$ momentum kinetic energy amount from Equation (11), neither of them takes into account the energy corresponding to mass increment $\Delta m_{m}$ from Equation (2), despite its proven existence experimentally confirmed by the Kaufman experiments [21] as 
measured by means of transverse interaction, and consequently, this is why neither paradigm assigns any function to the magnetic fields of charged particles nor to their magnetic mass increments in submicroscopic interaction.

This pinpoints exactly where the disconnect resides between classical/relativistic mechanics and quantum mechanics on one hand, and electromagnetic mechanics on the other hand, and reveals the importance of the adiabatic nature of energy induction [10] by means of the Coulomb interaction as shown with Figure 1 and Equation (20), that combines as Equation (13) the total amount of energy adiabatically induced in charged particles as calculated with Equation (11) for the translational momentum component, and with Equation (2) for the magnetic mass increment.

The critical disconnect resides precisely in the fact that the $\Delta K$ momentum kinetic energy half of the total energy quantum induced is adiabatically induced by the Coulomb interaction (Equation (12)) in such a way that it can only remain physically present and vectorially active in the axial direction of the proton, even if it is experimentally proven not to be able to cause the electron to move forward according to its vectorial direction toward the proton, nor along the trajectory mandated by classical mechanics since its vectorial orientation is immutably set by structure perpendicularly to this classical trajectory.

This brings to attention the fact that the relativistic momentum energy $\Delta K$ of Equation (6) and the $\Delta \mathrm{m}_{\mathrm{m}}$ relativistic mass increment from Equation (2) as combined in Equation (13), that completely account for the relativistic velocity and relativistic mass increase confirmed by the Kaufmann experiment [21], remain entirely adiabatically induced even when the related relativistic velocity is prevented by "something" from being expressed when the electron is stabilized in the hydrogen atom ground state.

This in turn leads to the conclusion that terms such as "electromagnetic momentum" and "magnetic mass increment" would be more appropriate than the current terms "relativistic momentum" and "relativistic mass increments" to describe these adiabatically induced energy half-quanta since it can be demonstrated that electromagnetic energy is adiabatically induced strictly as a function of the distance between charges, according to the induction growth curve dependent on the gamma factor and the Coulomb force [11] [21] [22], and that contrary to the very foundation of all traditional theories about energy and matter exclusively elaborated from macroscopic level experiments, according to which kinetic energy can exist only if translational motion is possible, kinetic energy is found, from all experiments involving submicroscopic charged elementary particles, to be a "physically existing substance" whose existence does not depend on velocity as currently axiomatically assumed, but that it is velocity that depends on the prior existence of kinetic energy, a velocity that can be expressed only if charged particles' translational motion is not impeded by local magnetic translational counter-pressure [10].

The ultimate question then turns out to be: How could this "something" operate to so effectively and so systematically hinder the natural motion of the $\Delta K$ 
momentum kinetic energy of the electron in such a way that it makes it impossible for it to go crashing onto the proton in accordance with its natural vectorial orientation?

Neither classical/relativistic mechanics nor quantum mechanics offer any mechanical clue to resolve this issue. But the trispatial geometry allows observing that this hindrance can only be provided by a predominantly repulsive magnetic interaction, that is, a magnetic counter-pressure, resulting from of the constant parallel/anti-parallel magnetic spins orientation switching interaction between the magnetic energy " $\mathrm{B}$ " of the electron invariant rest mass and those of the 3 quarks carrier-photons " $\mathrm{N}$ " of the proton [10] [11] [34], as symbolically represented with Figure 9 and Figure 10 that we will now analyze.

It must be clearly understood that it is the spherical increase/decrease motion of the physical presence of the actual "magnetic energy substance" of the electron and of the 3 quarks carrier-photons that must be visualized during this analysis, and not that of their $\boldsymbol{E}$ and $\boldsymbol{B}$ fields representations of Maxwell's equations, as we would be intuitively tempted to do.

To really understand the axial resonance trajectory that the electron is forced to move into, that determines the volume defined by the Schrödinger wave function, the relative powers of the oscillating magnetic spheres involved must be put in perspective.

In this process, the magnetic half-quantum $\Delta m_{m}$ of the electron carrier-photon "C" will be ignored to simplify the current analysis, because it is infinitesimal in the definition of the ground state resonance volume when compared to the role played by the magnetic mass " $\mathrm{B}$ " of the electron as revealed by its value calculated with Equation (27) and the following ratio established with the magnetic mass of the electron stabilized in the hydrogen ground state, and is significant only with respect to the transverse Zitterbewegung of the electron previously analyzed:

$$
\frac{\Delta m_{m}}{m_{e} / 2}=\frac{2.425337726 \mathrm{E}-35}{4.55469094 \mathrm{E}-31}=\frac{1}{1.877961527 \mathrm{E} 4}
$$

The $\Delta K$ momentum half-quantum " $\mathrm{F}$ " of the electron carrier-photon does have a role to play however, because each time that the magnetic sphere " $B$ " of the electron rest mass reduces to zero presence within $\mathrm{Z}$-space, all magnetic counter-pressure disappears by structure between the electron and the magnetic energy making up the magnetic spheres " $\mathrm{N}$ " centered on the proton, which causes the $\Delta K$ momentum energy " $F$ " of the electron to be free again to propel the electron toward the proton, until the magnetic energy substance " $B$ " of the electron rest mass begins to increase again in $\mathrm{Z}$-space as the following cycle of its frequency initiates.

On the side of the proton, it is the magnetic masses " $O$ " of the up and down quarks that will be ignored, because contrary to the insignificance of the magnetic $\Delta m_{m}$ half-quantum "C" of the electron carrier-photon with respect to the magnetic mass of the electron as shown with Equation (65), it is the magnetic masses "O" of the up and down quarks that are insignificant when compared to 
the immensely larger values of the $\Delta \mathrm{m}_{\mathrm{m}}$ magnetic half-quanta of their carrier-photons. Indeed, as calculated in reference [18] each quark carrier-photon "N" would have a mean total energy of about $310.457837 \mathrm{MeV}$ :

$$
\text { Quark carrier-photon energy }=\Delta K+\Delta m_{m}=4.974 \mathrm{E}-11 \mathrm{j}
$$

which sets their frequency and wavelength to the following values:

$$
v=\frac{E}{h}=7.506837869 \mathrm{E} 22 \mathrm{~Hz}, \lambda=\frac{c}{v}=3.993591752 \mathrm{E}-15 \mathrm{~m}
$$

and even without taking into account the magnetic drift caused by the so close mutual proximity of the 6 inner electron electromagnetic quanta of the proton (Ref: Equation (62) and references [18] [34]), that considerably increases their magnetic energy, to simplify this analysis, each of their $\Delta m_{m}$ magnetic half-quanta will minimally have the following value:

$$
\Delta m_{m}=\frac{E / 2}{c^{2}}=2.767206524 \mathrm{E}-20 \mathrm{~kg}
$$

In relation with the up quark mass available in Table 2, the following ratio can be established:

$$
\frac{\Delta m_{m}}{m_{U} / 2}=\frac{2.767206524 \mathrm{E}-20}{1.024805462 \mathrm{E}-30}=\frac{2.700226166 \mathrm{E} 10}{1}
$$

and for the down quark:

$$
\frac{\Delta m_{m}}{m_{D} / 2}=\frac{2.767206524 \mathrm{E}-20}{4.09922189 \mathrm{E}-30}=\frac{0.6750565347 \mathrm{E} 10}{1}
$$

So, comparing these last two ratios with the electron magnetic masses ratio calculated with Equation (65), not only do we observe that these two ratios are reversed with respect to the relation between the electron magnetic mass and that of its carrier-photon, but we also observe that the quarks carrier-photons are 10 orders of magnitude more energetic than the quarks that they carry, which justifies taking only the magnetic spheres " $\mathrm{N}$ " of these 3 carrier-photons into account to summarily explain the electron resonance volume.

Finally, the ratio of the magnetic mass " $\mathrm{B}$ " of the electron $m_{e} / 2$ with respect to the minimal $\Delta m_{m}$ magnetic mass " $\mathrm{N}$ " of even only one of the quarks' carrier-photons, will give a glimpse of how easily and strongly the electron can be set in resonance like a feather in hurricane winds as it is shoved about axially by even the minimal eleven-fold greater magnitude magnetic energy of the even only one quark carrier-photon centered on the proton location:

$$
\frac{m_{e} / 2}{\Delta m_{m}}=\frac{4.55469094 \mathrm{E}-31}{2.767206524 \mathrm{E}-20}=\frac{1.64595266 \mathrm{E} 11}{1}
$$

Facing the single electron oscillating magnetic energy sphere " $\mathrm{B}$ ", the combined magnetic energy of the inner proton components materialize as two relatively concentric antiparallel energy spheres of unequal spherical volumes (see Figure 10). The largest is made of the cyclically varying sum of the magnetic energy of two quarks carrier-photons $(2 \times$ "N") in permanent mutual parallel 
spins alignment as it cyclically increases and decreases between zero energy presence and maximum energy presence in Z-space, while the smallest magnetic sphere is made of the magnetic energy " $N$ " of the remaining third carrier-photon, which can only be by structure in antiparallel spin alignment with the first two, and whose energy is in constant oscillation in opposition with the sum of the spherical motion of the magnetic energy of the first two, that is, in increasing energy presence phase while the energy presence of the first two is decreasing, and in decreasing energy presence while the energy of the first two is increasing.

Additional legends for Figure 9, completing those defined for Figure 7 and Figure 8.

H. Symbolic representation of the resonance volume within which the electron point-like behaving trispatial junction and that of its carrier-photon remain captive in the hydrogen ground state orbital.

I. Proton.

J. Symbolic representation of the proton energy resonance volume, resulting from the cyclic spins reversal interaction between all 6 inner components of the proton structure, which are 2 up quarks, 1 down quark and their 3 carrier-photons.

K. Symbolic representation of the Zitterbewegung resonance volume within which the point-like behaving down quark trispatial junction and that of its carrier-photon involved in mutual cyclic magnetic spin reversal interaction within $\mathrm{Z}$-space remain captive, as represented with Figure 7 and Figure 8, but involving much higher frequencies than the Zitterbewegung resonance volume of the electron.

L. Symbolic representation of the Zitterbewegung resonance volume within which the point-like behaving up quark trispatial junction and that of its carrier-photon remain captive according to the same mechanics described for the down quark with previous representation $K$.

M. Hydrogen ground state mean orbital distance between the electron and the proton, corresponding to the theoretical Bohr radius at which the electron momentum energy is set at precisely $\Delta K$, outside of which distance this momentum energy diminishes to $\Delta K-\Delta(\Delta K)$ when the electron is pushed further away, and increases to $\Delta K+\Delta(\Delta K)$ when it is pulled closer to the proton, during its cyclic axial resonance motions sequences.

N. Symbolic representation of the spherical volume of the oscillating magnetic energy of a quark's carrier-photon in Z-space. Ref. Figure 5(c) as applied to the carrier-photon inner oscillating structure and Equation (55). This volume corresponds to its magnetic field varying from zero presence to a maximum presence that can be calculated with Equation (23), using the carrier-photon wavelength obtained with Equation (67), and to the velocity related quark magnetic mass increment $\Delta m_{m}$ obtained with Equation (68).

O. Symbolic maximum extent of the spherical volume of the oscillating magnetic energy of one quark up or down in Z-space. Ref. Figure 5(c) as applied to 
the quark inner oscillating structure and Equations (63) and (64).

Since all three quarks carrier-photons have the same frequency, they remain permanently synchronized in one of the two possible configurations, which are "U||U $\neq D$ " or "U ||$D \neq U$ " (see Figure 10).

The outcome is that irrespective of which increasing or decreasing presence phase of its magnetic oscillation "B" the electron may be in, either the larger or the smaller magnetic sphere centered on the proton location will be in parallel spin alignment with it and will repel it, which is what permanently interdicts that the electron could naturally reach the proton, unless having been accidentally or artificially induced from outside sources with a carrier-photon sufficiently energetic to do so, as is routinely done in high energy accelerators.

In the symbolic representation of Figure 9, the electron Zitterbewegung resonance volume " $\mathrm{A}$ " is oriented as if it was going to move toward the proton, to reflect the fact that the $13.6 \mathrm{eV}$ half-quantum of momentum energy $\Delta K$ re-induced in the electron carrier-photon as the electron was captured remains permanently oriented toward the proton, even if its forward motion is constantly inhibited by the fact that whatever increasing or decreasing presence phase its magnetic oscillating sphere may be in (Characteristic " $B$ " in Figure 7 and Figure 10), the latter will be repelled since either one or the other of the two mutually antiparallel magnetic spheres centered on the proton location (see Figure 10) will always be in repulsive parallel spin alignment with respect to the electron magnetic energy sphere " $B$ ".

This constant relative parallel repulsive spin orientation of the electron magnetic sphere with respect to at least one of the two proton concentric magnetic spheres is not however what explains the ground state orbital axial resonance state of the electron defined by the Schrödinger wave function. We will get into this in a moment, but let's first analyze the proton electromagnetic structure.

There may appear to be a disconnect between the idea that the energy of the magnetic oscillating spheres of the quarks carrier-photons could reach as far in space as the ground state orbital located at $5.29 \mathrm{E}-11 \mathrm{~m}$ from the proton, and with sufficient strength at that to establish a least action electromagnetic equilibrium state keeping the electron captive at this relatively great distance from the relatively minuscule volume of radius $1.2 \mathrm{E}-15 \mathrm{~m}$ within which we know that the 6 inner electromagnetic quanta making up the proton are captive into.

This is more easily put in perspective when considering that the magnetic field of the Sun reaches out as far as the outer limits of the Solar System, presumably as far as the Oort cloud, even if the matter of which the Sun is made is contained within a sphere whose radius is well shorter than the radius of Mercury's orbit, its innermost planet. Indeed, the huge magnetic field of the Sun can only be made of the sum of the individual magnetic fields of the innumerable elementary particles and carrier-photons making up the matter of which the Sun is made. The same conclusion can obviously be drawn for all existing celestial bodies, as put in perspective in reference [37]. 
So there is no disconnect between this conclusion drawn at the submicroscopic level and what can be observed even at the astronomic scale, because if a hydrogen atom was theoretically upsized sufficiently for its proton diameter to reach in dimension that of the Sun, then the electron would stabilize as far as Neptune's orbit, which relatively speaking, would give the magnetic field of the proton the same order of magnitude as that of the Sun.

Let us also recall that in the trispatial geometry, it is not within normal $\mathrm{X}$-space that this magnetic energy expands and contracts, but within magnetostatic Z-space, and that only the $\Delta \mathrm{K}$ momentum energy half-quanta and the point-like trispatial junctions $\otimes$ of each elementary particle, free moving photon and carrier-photon that actually really "live", so to speak, in normal X-space, that is, the only two aspects of elementary particle's electromagnetic energy that are physically detectable by means of frontal longitudinal collisions, which are the total sum of the electromagnetic energy that resides in the other two orthogonal spaces $\mathrm{Y}$ and $\mathrm{Z}$, and whose physical presence we can detect only through these trispatial junctions $\otimes$ that behave point-like in $\mathrm{X}$-space, and their $\Delta K$ translational momentum energy; and the only aspect of electromagnetic energy that can be detected by transverse collision or interaction, which is only the electromagnetic energy that resides in the other two orthogonal spaces and that we detect through these trispatial junctions $\otimes$ always behaving point-like in $\mathrm{X}$-space, that is, the energy of the rest masses " $m$ " of the electron, the positron, the up quark and the down quark, and the energy of the magnetic mass increments " $\Delta m_{m}$ " of the carrier-photons, and finally the electromagnetic energy half-quanta " $\Delta m_{m}$ " of freely moving photons.

Indeed, it is not the magnetic fields of the proton 6 inner components that are captive within its physically measured volume, but the 6 point-like behaving trispatial junctions $\otimes$ that are the individual anchoring locations of this magnetic energy within normal X-space, and through which their electromagnetic energy cyclically oscillates, that are captive by pairs in Zitterbewegung transverse resonance states, and also collectively in the common least action resonance volume resulting from their mutual trispatial electromagnetic interaction resulting in the establishment of the stable proton structure.

The neutron, which is not illustrated in this document, has an inner electromagnetic structure involving the same up and down quarks and their carrier-photons of slightly higher energy, with the difference that instead of involving 2 up quarks and 1 down quark (uud), it involves 2 down quarks and 1 up quark (udd). The details of the trispatial structures of both nucleons are available in reference [18].

With regard to the ground state orbital resonance volume, Figure 10 puts in perspective the fact that this resonance volume is due to the hugely higher oscillating frequency of the quarks carrying-photons energy with respect to the much slower oscillating frequency of the electron rest mass magnetic energy.

Relating these frequencies of the electron from Equation (15) and of a quark carrier-photon from Equation (67) allows determining that minimally, the 
magnetic polarity reversal of each quark carrier-photon occurs in excess of 600 times during each occurrence of magnetic polarity reversal of the electron magnetic energy, that is, during each magnetic presence cycle of the electron magnetic energy in Z-space:

$$
\frac{v_{\text {quark carrier-photon }}}{v_{\text {electron }}}=\frac{7.506837869 \mathrm{E} 22}{1.235589976 \mathrm{E} 20}=\frac{607.5508878}{1}
$$

The constant interplay due to the frequencies difference of the various magnetic spheres involving the inverse cube interaction law with distance, that opposes the $\Delta \mathrm{K}$ unidirectional momentum energy " $F$ " that constantly tends to propel the electron toward the proton, to an uninterrupted sequence of magnetic attraction-repulsion phases, can then only result in the establishment of the stable axial resonance state that de Broglie identified [1].

In Figure 10, the central sequence "B" symbolically represents an arbitrary sample of 6 occurrences of the intensity variation of the spherical presence of the electron magnetic energy as a function of its frequency. In a simplified manner, each of these 6 occurrences is confronted in the lower sequence by the more than 600 occurrences of the intensity variation of the spherical presence of the magnetic energy of the 3 carrier-photons of the up or down quarks of the proton as a function of their own frequencies.

The least action orbital equilibrium state is consequently established by the fact that the $\Delta \mathrm{K}$ momentum energy " $F$ " half-quantum of the electron carrier-photon, is alternately hindered in its forward motion, when the magnetic interaction function of the inverse cube law becomes repulsive-parallel magnetic spin alignment between the magnetic energy spheres of the electron and one of the proton magnetic energy spheres-and is then freed from this counter-pressure while the magnetic interaction becomes attractive-antiparallel magnetic spin alignment between the magnetic spheres involved.

As represented with Figure 10, during each of the 600 magnetic cycles of a quark's carrier-photon " $N$ ", the electron magnetic sphere " $\mathrm{B}$ " will be axially repelled away from the proton by distance " $\Delta d$ " during half of the carrier-photon "N" magnetic presence cycle, during which their spin alignment is parallel-thus repulsive, and since the electron will be farther away from the proton as the relation becomes antiparallel for the same duration, there will be a physical impossibility for it to be axially brought back all the way to distance " $-\Delta d$ ", given that the inverse cube force will be weaker at this farther location from the proton at the beginning of the antiparallel phase.

Therefore, and by structure, given the more weakly acting inverse cube attraction at the beginning of attractive phase, the electron can be axially brought back only to distance " $-(\Delta d-\Delta(\Delta d))$ ", which will cause it to progressively move away from the proton at each "B" $/$ "N" relative magnetic spins polarity reversal sequence until its own magnetic energy presence " $B$ " falls to zero, moment during which only the electron carrier-photon " $\Delta K$ " half-quantum momentum energy will be active, now causing the electron to freely move as close to the 
proton as the Coulomb force inverse square law will bring it, until its next magnetic presence cycle " $\mathrm{B}$ " initiates and that the whole predominantly repulsive magnetic sequence "B"/"N" is initiated again, as represented with Figure 10.

Of course the actual resonance state of the electron in the least action orbital of the hydrogen atom or in any other atom will be much more complex than hinted at with this limited example, which is only meant to describe the fundamental mechanics of the magnetic interaction between the electron magnetic energy " $B$ " and " $\Delta K$ " momentum energy on one hand, and the magnetic energy of the quarks carrier-photons of the proton, on the other hand. Obviously, the exact resonance volume within which each elementary electromagnetic massive particle in the hydrogen atom will be circumscribed, which are one electron, one down quark and two up quarks, can eventually be determined only by a careful study of all electromagnetic interactions between them and their carrier-photons.

Given that the mean equilibrium distance that this process forces the electron in motion to stabilize at in the hydrogen atom coincides with the densest area of probability distribution of Heisenberg's statistical method, it would seem that the axial trajectory of the electron about this mean distance within the volume that the electron can thus visit as a function of its varying relativistic mass and related inertia at any given instant, it should directly correspond with Heisenberg's probability distribution of all of the possible instantaneous locations that the electron can be stochastically calculated to be localized at when repeatedly theoretically collapsing the wave function in its current form [5] [21], and whose quantized axial beat can no doubt be related to the regularities of the fine structure of the hydrogen spectrum, that Sommerfeld first associated to a hypothetical elliptical orbit that the electron would follow, in his attempt to explain the fine splitting of the main spectral lines ([36], p. 114).

So the very limited resonance volume in $\mathrm{X}$-space within which the $\Delta K$ momentum and the trispatial junctions $\otimes$ of a moving electron will be localized within can be represented as:

$$
\int_{-d}^{+d}|\psi|^{2} \mathrm{~d} x \mathrm{~d} y \mathrm{~d} z=1
$$

while the theoretical resonance volume in Z-space within which the magnetic energy of the same moving electron can be represented would be:

$$
\int_{-\infty}^{+\infty}|\psi|^{2} \mathrm{~d} x \mathrm{~d} y \mathrm{~d} z=1
$$

It seems also entirely reasonable to conclude that the elementary charged up and down quarks making up the scatterable inner structure of protons and neutrons and their carrier-photons, which are known to be the only existing elementary electromagnetic subcomponents of all atomic nuclei, as analyzed in reference [21], should be subject to similar resonance states within their own local least action electromagnetic equilibrium states, that could then also potentially be described by the various methods of quantum mechanics in a manner 
more satisfactory than quantum chromodynamics (QCD) has achieved.

\section{Conclusions}

Although not providing the progressive mechanical explanation of the transitions between stationary states that de Broglie and Schrödinger were working to resolve, this article proposes an electromagnetic resonance mechanics of elementary electromagnetic particles that, if confirmed, could possibly allow it.

Unexpectedly, this mechanics also brings to light the possibility of using the various methods of quantum mechanics to establish wave functions to describe the resonance states of the elementary particles making up the inner scatterable structure of protons and neutrons.

This mechanics of establishment of the resonance volume represented by a wave function is grounded on the identification of the anchoring points $\otimes$ inside this volume of both quanta of electromagnetic energy constituting an elementary electromagnetic particle, that is, the trispatial junctions through which the quanta of kinetic energy involved oscillate harmonically to establish this volume.

The trispatial geometry reveals that each stable elementary electromagnetic particle involves in fact a pair of separate electromagnetic quanta, that is, a massive stable elementary electromagnetic quantum which is intrinsically translationally inert in X-space-electron, positron, up quark and down quark-possessing a measurable electric charge and a measurable magnetic field, accompanied by a carrier-photon, possessing a pair of electric charges whose opposite signs mutually cancel each other and a measurable magnetic field, and which contributes the momentum $\Delta K$ of the inert quantum that it accompanies in space, as well as its electromagnetic mass increment $\Delta m_{m}$.

This geometry reveals furthermore that the spin of elementary particles is a property of relative alignment of magnetic polarity between the electromagnetic particles and not an intrinsic property of angular moment of these particles, and that the half-quantum of magnetic energy of any electromagnetic quantum oscillates between a state of maximum presence and a state of zero presence in $\mathrm{Z}$-space at the frequency of its energy.

Finally, the trispatiale geometry reveals that it is the differences of frequencies of oscillation of the half-quanta of magnetic energy of elementary quanta that explain the stability of all least action orbitals in atoms, as well as all of their resonance states.

\section{References}

[1] De Broglie, L. (1923) Comptes rendus T., 177, 507-510. http://www.academie-sciences.fr/pdf/dossiers/Broglie/Broglie_pdf/CR1923_p507.pdf

[2] Einstein, A., Schrödinger, E., Pauli, W., Rosenfeld, L., Born, M., Joliot-Curie, I. \& F., Heisenberg, W., Yukawa, H., et al. (1953) Louis de Broglie, physicien et penseur. Éditions Albin Michel, Paris.

[3] De Broglie, L. (1924) Comptes rendus de l'Académie des Sciences (Paris), 179, 39.

[4] De Broglie, L. (1924) Comptes rendus de P Académie des Sciences (Paris), 179, 676. 
[5] Schrödinger, E. (1952) British Journal for the Philosophy of Science, 3, 109-233. https://doi.org/10.1093/bjps/III.10.109

[6] Golovko, V.A. (2008) Electromagnetic Radiation and Resonance Phenomena in Quantum Mechanics. arXiv:0810.3773v2

[7] Schrödinger, E. (1930) Über die kräftefreie Bewegung in der relativistischen Quantenmechanik. Sitzungsberichte Akad. Berlin, 418-428.

[8] Michaud, A. (2016) Journal of Physical Mathematics, 7, 153.

https://www.omicsonline.com/open-access/on-de-broglies-doubleparticle-photon-h ypothesis-2090-0902-1000153.pdf https://doi.org/10.4172/2090-0902.1000153

[9] Marmet, P. (2003) International IFNA-ANS Journal, 9. 64-76. http://www.newtonphysics.on.ca/magnetic/index.html

[10] Michaud, A. (2016) Journal of Physical Mathematics, 7, 177. http://www.omicsonline.com/open-access/on-adiabatic-processes-at-the-elementar y-particle-level-2090-0902-1000177.php?aid=75602

[11] Michaud, A. (2017) Journal of Astrophysics \& Aerospace Technology, 5, 152. https://www.omicsonline.org/open-access/gravitation-quantum-mechanics-and-the -least-action-electromagneticequilibrium-states-2329-6542-1000152.pdf

[12] Michaud, A. (2017) Journal of Physical Mathematics, 8, 217. https://www.omicsonline.com/open-access/the-last-challenge-of-modern-physics- 2 090-0902-1000217.pdf

[13] Michaud, A. (2013) International Journal of Engineering Research and Development, 7, 32-39. http://ijerd.com/paper/vol7-issue4/G0704032039.pdf

[14] Michaud, A. (2007) International IFNA-ANS Journal, 13, 123-140. http://www.gsjournal.net/Science-Journals/Essays/View/2257

[15] Michaud, A. (2013) International Journal of Engineering Research and Development, 6, 31-45. http://ijerd.com/paper/vol6-issue8/G06083145.pdf

[16] Michaud, A. (2013) International Journal of Engineering Research and Development, 7, 1-8. http://www.ijerd.com/paper/vol7-issue7/A07070108.pdf

[17] Michaud, A. (2013) International Journal of Engineering Research and Development, 6, 1-10. http://ijerd.com/paper/vol6-issue10/F06103649.pdf

[18] Michaud, A. (2013) International Journal of Engineering Research and Development, 7, 29-53. http://ijerd.com/paper/vol7-issue9/E0709029053.pdf

[19] Michaud, A. (2013) International Journal of Engineering Research and Development, 6, 27-34. http://www.ijerd.com/paper/vol6-issue6/F06062734.pdf

[20] Sears, F., Zemansky, M. and Young, H. (1984) University Physics. 6th Edition, Addison Wesley, Boston.

[21] Kaufmann, W. (1903) Über die "Elektromagnetische Masse" der Elektronen, Kgl. Gesellschaft der Wissenschaften Nachrichten. In: Mathematisch-Physikalische Klasse, Vandenhoeck \& Ruprecht, Deutsch, 91-103.

http://gdz.sub.uni-goettingen.de/dms/load/img/?PPN=PPN252457811_1903\&DMD ID=DMDLOG_0025

[22] Michaud, A. (2013) International Journal of Engineering Research and Development, 6, 1-10. http://www.gsjournal.net/Science-Journals/Essays/View/3197

[23] Michaud, A. (2013) International Journal of Engineering Research and Development, 7, 21-25. http://ijerd.com/paper/vol7-issue3/E0703021025.pdf

[24] Schwinger, J. (1948) Physical Review, 73, 416-417. 
https://doi.org/10.1103/PhysRev.73.416

[25] Haskell, R.E. (2003) Special Relativity and Maxwell's Equations. http://richardhaskell.com/files/Special\%20Relativity\%20and\%20Maxwells\%20Equat ions.pdf

[26] Ernst, A. and Hsu, J.P. (2001) Chinese Journal of Physics, 39. http://adsabs.harvard.edu/cgi-bin/nph-data_query?bibcode=2001ChJPh..39..211E\&l ink_type $=$ ARTICLE\&db_key=PHY\&high $=$

[27] Michaud, A. (2017) Electromagnetic Mechanics of Elementary Particles. 2nd Edition, Scholar's Press, Saarbrücken.

Https://www.morebooks.de/store/gb/book/electromagnetic-mechanics-of-elementa ry-particles/isbn/978-3-330-65345-0

[28] De Broglie, L. (1993) La physique nouvelle et les quanta. 2nd Edition, Flammarion, France.

[29] Michaud, A. (2000) On an Expanded Maxwellian Geometry of Space. Proceedings of Congress-2000-Fundamental Problems of Natural Sciences and Engineering, Volume 1, St. Petersburg, 291-310.

[30] Michaud, A. (2013) International Journal of Engineering Research and Development, 6, 7-11. http://ijerd.com/paper/vol6-issue12/B06120711.pdf

[31] Breidenbach, M., et al. (1969) Physical Review Letters, 23, 935-939. http://www.slac.stanford.edu/pubs/slacpubs/0500/slac-pub-0650.pdf https://doi.org/10.1103/PhysRevLett.23.935

[32] Particle Data Group (2000) The European Physical Journal-Review of Particle Physics, 15, 4 .

[33] Kotler, S., Akerman, N., Navon, N., Glickman, Y. and Ozeri, R. (2014) Nature Magazine, 510, 376-380.

http://www.nature.com/articles/nature13403.epdf?referrer_access_token=yoC6RXrPyx wvQviChYrG0tRgN0jAjWel9jnR3ZoTv0PdPJ4geER1fKVR1YXH8GThqECstdb6e48m Zm0qQo2OMX_XYURkzBSUZCrxM8VipvnG8FofxB39P4lc-1UIKEO1

[34] Michaud, A. (2013) International Journal of Engineering Research and Development, 7, 50-66. http://www.ijerd.com/paper/vol7-issue5/H0705050066.pdf

[35] Feynman, R.P., Leighton, R.B. and Sands, M. (1964) The Feynman Lectures on Physics. Addison-Wesley, Boston, Vol. II, 28-1. https://doi.org/10.1063/1.3051743

[36] Eisberg, R. and Resnick, R. (1985) Quantum Physics of Atoms, Molecules, Solids, Nuclei, and Particles. 2nd Edition, John Wiley \& Sons, New York.

[37] Michaud, A. (2013) International Journal of Engineering Research and Development, 8, 10-33. http://ijerd.com/paper/vol8-issue1/B08011033.pdf 